\title{
A Mach-sensitive implicit-explicit scheme adapted to compressible multi-scale flows
}

\author{
D. Iampietro ${ }^{\mathrm{a}, \mathrm{b}, \mathrm{e}, *}$, F. Daude ${ }^{\mathrm{a}, \mathrm{b}}$, P. Galon ${ }^{\mathrm{b}, \mathrm{c}}$, J.-M. Hérard $^{\mathrm{d}, \mathrm{e}}$ \\ ${ }^{a}$ EDF RED, Département ERMES, 7 Boulevard Gaspard Monge, 91120 Palaiseau, France \\ ${ }^{b}$ IMSIA, UMR EDF/CNRS/CEA/ENSTA 9219, Université Paris-Saclay, 828 Boulevard des Maréchaux, 91762 Palaiseau Cedex, France \\ ${ }^{c} C E A, D E N, D A N S, D M 2 S$, SEMT, DYN, F-91191 Gif-sur-Yvette, France \\ ${ }^{d}$ EDF RED D, Département MFEE, 6 Quai Watier, F-78401 Chatou, France \\ ${ }^{e}$ I2M, UMR CNRS 7373, Technopôle Château-Gombert, 39 rue F. Joliot Curie, F-13453 Marseille, France
}

\begin{abstract}
The method presented below focuses on the numerical approximation of the Euler compressible system. It pursues a two-fold objective: being able to accurately follow slow material waves as well as strong shock waves in the context of low Mach number flows. The resulting implicit-explicit fractional step approach leans on a dynamic splitting designed to react to the time fluctuations of the maximal flow Mach number. When the latter rises suddenly, the IMEX scheme, so far driven by a material-wave Courant number, turn into a time-explicit approximate Riemann solver constrained by an acoustic-wave Courant number. It is also possible to enrich the dynamic splitting in order to capture high pressure jumps even when the flow Mach number is low. One-dimensional low Mach number test cases involving single or multiple waves confirm that the present approach is as accurate and efficient as an IMEX Lagrange-Projection method. Besides, numerical results suggest that the stability of the present method holds for any Mach number if the Courant number related to the convective subsystem arising from the splitting is of order unity.
\end{abstract}

Keywords: Fractional Step, Implicit-Explicit Schemes, Multi-scale Flows, Low Mach Number, Relaxation Schemes, Hyperbolic Equations

\section{Introduction}

The present work deals with the construction of a time implicit-explicit scheme providing a sketch of answer to cope with multi-scale wave scenarios and more specifically with what is called a condensation induced water hammer (CIWH).

Indeed, in the very first instants of this phenomenon, one is interested in following a slow interface between hot vapor and cooler liquid water. Since the speed of such a material wave is of the order of $1 \mathrm{~m} . \mathrm{s}^{-1}$, which is considerably smaller than the acoustic wave speeds in both phases, the interface dynamics is typical of low Mach number flows. Nonetheless, as time goes on, shear instabilities and steep temperature gradients entail the trapping and then the sudden condensation of vapor pockets. This leads finally to the production of strong shock waves in the liquid phase. The objective is thus to design a numerical scheme accurate for material waves in a low Mach number flow while being able to capture high pressure gradients.

On one hand, fulfilling both aims might seem contradictory if one considers the Euler or Navier-Stokes incompressible systems since their divergence-free constraint prohibits any compressible effects and hence the occurrence of compressive shock waves. On the other hand, the pioneering works of Joukowski [1] and Allievi [2] state that, at constant temperature, pressure jumps in a low Mach number compressible flow are given by: $\Delta p=\rho^{0} c^{0} \Delta u$; with $\rho^{0}$ (respectively $c^{0}$ ) the constant density (respectively the constant speed of sound) of the fluid. See also [3] for a review of the water hammer theory. Thus, in the case of liquid water, at $295 \mathrm{~K}, \rho^{0} \approx 10^{3} \mathrm{~kg} \cdot \mathrm{m}^{-3}, \mathrm{c}^{0} \approx 1.5 \times 10^{3} \mathrm{~m} \cdot \mathrm{s}^{-1}$. If one assumes

*Corresponding author: david.iampietro@edf.fr 
that $\Delta u \approx 1 \mathrm{~m} \cdot \mathrm{s}^{-1}$, pressure jumps amplitude is of $15 \mathrm{bar}$ which is the order of magnitude observed experimentally in [4].

From a numerical point of view, different strategies have been adopted in order to be accurate on slow material waves in the case of low Mach number flows. Preconditioning methods stemming from [5] and improved in [6, 7, 8] aim at modifying the Jacobian eigenvalues of hyperbolic systems in order to get rid of their constraining acoustic part. Asymptotic preserving schemes (AP schemes), introduced by Jin in [9], are based on the identification of a non-stiff and a stiff part of hyperbolic systems. The latter is then discretized using a time-implicit method which allows the scheme to be consistent, for fixed time and space-steps, with a targeted asymptotic discrete solver as a scale parameter tends towards zero. Recently in [10, 11, 12, 13], different kinds of AP schemes have been derived to seize the incompressible limit of the Euler or the Navier-Stokes system as the flow Mach number tends towards zero. It results in an implicit-explicit (IMEX) algorithm providing a time-implicit discretization for the hydrodynamic pressure gradient, and a time-explicit discretization for the convective terms. Besides, if one considers the Courant number $\mathscr{C}$ based on the slowest material waves of the flow, a key property for the above AP schemes is also to remain stable for $\mathscr{C} \approx 1$ at any Mach number. This typical issue has been explored using the "modified equation" tools as well as the spectral theory by Noelle an his collaborators in [14, 15]. See also [16] in which the AP property as well as the Mach-uniform stability property has been proved for a Lagrange-Projection method described in [17].

If the Mach number flow is small, the above strategies produce satisfying results when one seeks to seize the slow material waves dynamics as well as the pressure incompressible profile. However, no satisfying solution has been yet found in order to dynamically capture strong shock waves if they suddenly appear in such a configuration.

Continuing ideas suggested in [18, 19, 20], the present work derives an IMEX scheme based on a Mach-sensitive splitting of the Euler system. Such a splitting stems from the pioneering work of Baraille et al. [21] extended by Buffard et al. [22]. Contrary to [21, 13, 22, 17], the splitting evolves dynamically in time thanks to a parameter measuring a priori the instantaneous maximal Mach number of the flow. It results in a dynamically Weighted Fractional Step Approach (WFSA) enabling to cope with a wide panel of situations. Indeed, in the context of a low Mach number flow, starting from a completely decoupled IMEX formulation with $\mathscr{C} \approx 1$, it offers the possibility to retrieve a time-explicit Godunov-like solver for the overall Euler system if a sudden rise of the Mach number is detected. The CFL condition adapts itself and is re-based on the fastest physically relevant wave speed, i.e. the acoustic one. Thus, if this rise of the Mach number is associated with the production of shock waves, they will be optimally captured. What is more, the temporal splitting parameter can be enriched by a simple "shock detector" ensuring that a time-explicit solver is recovered when high amplitude shock waves arise even if the material velocity is low. Then, strong water hammer pressure jumps occurring in low Mach number flows can also be handled.

The second section of this article is a condensed presentation of the Mach-sensitive splitting fully described in [19, 20]. The readers are notably referred to this work for the construction of a time-explicit scheme for both convective and acoustic parts of the Mach-sensitive splitting. Hence, the third and the fourth section of the present manuscript are entirely dedicated to the study and then the derivation of a time-implicit scheme related to the acoustic part of the splitting. It leans on the relaxation schemes theory and particularly on a Suliciu-like relaxation procedure detailed in [23, 24, 18, 25]. The fifth section aggregates four types of one-dimensional numerical results. A first part briefly describes the effect of the Courant number when an IMEX scheme is triggered on isolated shock or contact waves in the context of a low Mach number flow. Secondly, the accuracy and the efficiency of the present approach are compared with the Lagrange-Projection fractional step method described in [17]. This comparative study is based on a low-Mach shock tube test case in which pressure fluctuations remain small. Thirdly a double Riemann problem involving a stiffened gas equation of state is examined. It aims at modeling the occurrence of water hammers in a low-Mach number flow. Finally, in the last part of the numerical results, the ability of the proposed IMEX scheme to deal with very specific low-velocity flow regimes is assessed. It focuses on the capture of constant states initially perturbed by small amplitude acoustic waves as well as the capacity to compute weakly compressible approximate solutions. 


\section{A Mach-Sensitive Fractional Step Approach}

The present work focuses on the Euler compressible system. However its extension to the two-phase Homogeneous Equilibrium Model (HEM, [26]) in which both phases have the same velocity $\mathbf{u}$, pressure $p$, temperature $T$ and chemical potential is straightforward since it results in the same conservation law structure, namely:

$$
\begin{aligned}
& \partial_{t} \rho+\operatorname{div}(\rho \mathbf{u})=0, \\
& \partial_{t}(\rho \mathbf{u})+\operatorname{div}(\rho \mathbf{u} \otimes \mathbf{u}+p \mathbf{I})=\mathbf{0}, \\
& \partial_{t}(\rho e)+\operatorname{div}((\rho e+p) \mathbf{u})=0, \\
& e=\frac{|\mathbf{u}|^{2}}{2}+\varepsilon, \varepsilon=\varepsilon^{E O S}(\rho, p), \\
& (\rho c)^{2}=\left(\partial_{p} \varepsilon_{\mid \rho}\right)^{-1}\left(p-\rho^{2} \partial_{\rho} \varepsilon_{\mid p}\right),
\end{aligned}
$$

with $\rho$ the density of the mixture and $\varepsilon$ its specific internal energy. The function $\varepsilon^{E O S}(.,$.$) defines the equation of state$ between the thermodynamic variables while $c$ is the sound speed involved in the nonlinear wave propagation.

As described in [19, 20], it is possible to derive a scheme able to deal with highly compressible flows as well as low . Mach number flows by splitting the system 2.1$]$ into a convective $(C)$ and an acoustic $(\mathcal{A})$ subsystem:

$$
C:\left\{\begin{array}{l}
\partial_{t} \rho+\operatorname{div}(\rho \mathbf{u})=0, \\
\partial_{t}(\rho \mathbf{u})+\operatorname{div}\left(\rho \mathbf{u} \otimes \mathbf{u}+\mathscr{E}_{0}^{2}(t) p \mathbf{I}\right)=\mathbf{0}, \\
\left.\partial_{t}(\rho e)+\operatorname{div}\left((\rho e)+\mathscr{E}_{0}^{2}(t) p\right) \mathbf{u}\right)=0,
\end{array}\right.
$$

$$
\mathcal{A}:\left\{\begin{array}{l}
\partial_{t} \rho=0, \\
\partial_{t}(\rho \mathbf{u})+\operatorname{div}\left(\left(1-\mathscr{E}_{0}^{2}(t)\right) p \mathbf{I}\right)=\mathbf{0}, \\
\partial_{t}(\rho e)+\operatorname{div}\left(\left(1-\mathscr{E}_{0}^{2}(t)\right) p \mathbf{u}\right)=0 .
\end{array}\right.
$$

Here, $\left.\left.\mathscr{E}_{0}(t) \in\right] 0,1\right]$ is a dynamic splitting parameter designed to be equal to one in the case of sonic or supersonic flows or equal to the maximal flow Mach number in the case of subsonic flows:

$$
\begin{aligned}
& \mathscr{E}_{0}(t)=\max \left(M_{\text {inf }}, \min \left(M_{\max }(t), 1\right)\right), \\
& M_{\text {max }}(t)=\sup _{x \in \Omega}\left(M(x, t)=\frac{|u(x, t)|}{c(x, t)}\right),
\end{aligned}
$$

with $M_{\text {inf }}$ a given lower bound preventing $\mathscr{E}_{0}(t)$ from being exactly equal to zero. As shown in [19, 20], both subsystems are hyperbolic for a stiffened gas EOS provided that the pressure remains positive throughout space and time. In one dimension, their eigenvalues are:

$$
\begin{array}{ll}
\lambda_{1}^{C}=u-\mathscr{E}_{0} c_{C} & \leq \lambda_{2}^{C}=u \leq \lambda_{3}^{C}=u+\mathscr{E}_{0} c_{\mathcal{C}}, \\
\lambda_{1}^{\mathcal{A}}=-\left(1-\mathscr{E}_{0}^{2}\right) c_{\mathcal{A}} & \leq \lambda_{2}^{\mathcal{A}}=0 \leq \lambda_{3}^{\mathcal{A}}=\left(1-\mathscr{E}_{0}^{2}\right) c_{\mathcal{H}},
\end{array}
$$

with $c_{\mathcal{C}}$ (respectively $c_{\mathcal{A}}$ ) the convective (respectively the acoustic) celerity defined by:

$$
\begin{aligned}
& \left(\rho c_{C}(\rho, p)\right)^{2}=\left(\partial_{p} \varepsilon_{\mid \rho}\right)^{-1}\left(\mathscr{E}_{0}^{2} p-\rho^{2} \partial_{\rho} \varepsilon_{\mid p}\right), \\
& \left(\rho c_{\mathcal{A}}(\rho, p)\right)^{2}=\left(\partial_{p} \varepsilon_{\mid \rho}\right)^{-1} p,
\end{aligned}
$$

and:

$$
\left(c_{C}\right)^{2}+\left(1-\mathscr{E}_{0}^{2}\right)\left(c_{\mathcal{H}}\right)^{2}=c^{2} .
$$

What is more, for both subsystems, the 1-wave and 3-wave are associated to genuinely non-linear fields whereas the 2-wave field is linearly degenerate.

It can be noticed that, when the Mach number is small so that $\mathscr{E}_{0}(t)$ is close to zero, pressure terms completely disappear from the subsystem $C$ which only conserves the convective spatial operator div $(\mathbf{u} *)$. Pressure terms are retrieved in the subsystem $\mathcal{A}$ which turns out to hold most of the acoustic effects. That is why, in the case of low Mach number flows, if the physics of interest is essentially related to material waves propagating at speed $|u| \ll c$, a time-implicit discretization will be provided for subsystem $\mathcal{A}$ while $C$ will be solved with a time-explicit scheme. 
Indeed, define $\Delta x$ the space-step of the computational domain. If $\Delta t$ is the time-step guaranteeing the stability of the numerical scheme, one can formally introduce several Courant numbers related to the above wave speeds, namely:

$$
\begin{array}{lr}
\mathscr{C}_{E}=\frac{\Delta t}{\Delta x}(|u|+c) & \text { Euler Courant number, } \\
\mathscr{C}_{|u|}=\frac{\Delta t}{\Delta x}|u| & \text { Convective Courant number, } \\
\mathscr{C}_{C}=\frac{\Delta t}{\Delta x}\left(|u|+\mathscr{E}_{0} c_{C}\right) & \text { Courant number related to } C, \\
\mathscr{C}_{\mathcal{A}}=\frac{\Delta t}{\Delta x}\left(\left(1-\mathscr{E}_{0}^{2}\right) c_{\mathcal{A}}\right) & \text { Courant number related to } \mathcal{A} .
\end{array}
$$

By using a time-implicit scheme for the resolution of the subsystem $\mathcal{A}$, one seeks to be relieved from most of the time-explicit stability condition: $\mathscr{C}_{E}<1$. Particularly, if the scheme allows to fulfill $\mathscr{C}_{C} \approx 1$ when $\mathscr{E}_{0} \ll 1$, one expects a substantial drop of the numerical diffusion holding on the material waves propagating at speed $|u|$. We refer the readers to [19, 20] for the complete study of both continuous subsystems $C$ and $\mathcal{A}$ as well as the derivation of $a$ full time-explicit fractional step involving relaxation schemes. A short description of the time-explicit convective flux related to the subsystem $C$ is written in Appendix A.

In the following, the derivation of the $\mathcal{A}$-time-implicit $\mathcal{C}$-time-explicit fractional step approach is presented. As the $\mathcal{C}$-time-explicit scheme described in [19, 20] is given in Appendix A focus is only given on the $\mathcal{A}$-time-implicit integration.

\section{A Sulicu-like Relaxation Scheme for the Acoustic Subsystem}

Let us introduce a new Suliciu-like relaxation subsystem $\mathcal{A}^{\mu}$ as:

$$
\begin{gathered}
\mathcal{A}^{\mu}:\left\{\begin{array}{l}
\partial_{t} \rho=0, \\
\partial_{t}(\rho u)+\partial_{x}\left(\left(1-\mathscr{E}_{0}^{2}(t)\right) \Pi\right)=0, \\
\partial_{t}(\rho \Pi)+\partial_{x}\left(\left(1-\mathscr{E}_{0}^{2}(t)\right) a_{\mathcal{A}}^{2} u\right)=\frac{\rho(p-\Pi)}{\mu}, \\
\partial_{t}(\rho e)+\partial_{x}\left(\left(1-\mathscr{E}_{0}^{2}(t)\right) \Pi u\right)=0,
\end{array}\right. \\
\left(\mathcal{A}^{\mu}\right)^{N C}:\left\{\begin{array}{l}
\partial_{t} \tau=0, \\
\partial_{t} u+\left(1-\mathscr{E}_{0}^{2}(t)\right) \tau \partial_{x} \Pi=0, \\
\partial_{t} \Pi+\left(1-\mathscr{E}_{0}^{2}(t)\right) a_{\mathcal{A}}^{2} \tau \partial_{x} u=\frac{(p-\Pi)}{\mu} \\
\partial_{t} e+\left(1-\mathscr{E}_{0}^{2}(t)\right) \tau \partial_{x}(\Pi u)=0 .
\end{array}\right.
\end{gathered}
$$

More details on relaxation schemes can be found in [23, 27, 25]. Moreover, the derivation of the above relaxation subsystem can be found in [19,20]. Recall that $\Pi$ is the relaxation pressure forced to converge towards the real pressure $p$ thanks to a source term of timescale $\mu \ll 1$. Besides, $a_{\mathcal{A}}$ is a relaxation constant encapsulating the thermodynamic nonlinearity. In order to provide sufficient diffusion to the relaxation subsystem, one can exhibit (see [28, 17, 19, 20]) the following subcharacteristic condition:

$$
a_{\mathcal{A}}>\rho c_{\mathcal{A}} .
$$

Let us define $\tau=1 / \rho$ the specific volume. Using the fact that the density is independent of time, the relaxation subsystem $\mathcal{A}^{\mu}$ is equivalent to its non-conservative version $\left(\mathcal{A}^{\mu}\right)^{N C}$. Then, one can easily prove that the relaxation subsystem is hyperbolic, and that its eigenvalues are:

$$
\lambda_{1}^{\mathcal{A}, \mu}=-\left(1-\mathscr{E}_{0}^{2}\right) a_{\mathcal{A}} \tau<\lambda_{2}^{\mathcal{A}, \mu}=\lambda_{3}^{\mathcal{A}, \mu}=0<\lambda_{4}^{\mathcal{A}, \mu}=\left(1-\mathscr{E}_{0}^{2}\right) a_{\mathcal{A}} \tau
$$


Besides all its characteristic fields are linearly degenerate. Let us now introduce $W$ and $R$ as:

$$
\begin{aligned}
W & =u-\Pi / a_{\mathcal{A}}, \\
R & =u+\Pi / a_{\mathcal{A}} .
\end{aligned}
$$

It is worth noticing that the non-conservative subsystem $\left(\mathcal{A}^{\mu}\right)^{N C}$ is equivalent to:

$$
\left\{\begin{array}{l}
\partial_{t} \tau=0, \\
\partial_{t} W+\lambda_{1}^{\mathcal{A}, \mu} \partial_{x} W=-\frac{(p-\Pi)}{a_{\mathcal{A}} \mu} \\
\partial_{t} R+\lambda_{4}^{\mathcal{A}, \mu} \partial_{x} R=\frac{(p-\Pi)}{a_{\mathcal{A}} \mu} \\
\partial_{t} e+\left(1-\mathscr{E}_{0}^{2}(t)\right) \tau \partial_{x}(\Pi u)=0
\end{array}\right.
$$

with $u(W, R)=(R+W) / 2$ and $\Pi(W, R)=a_{\mathcal{A}}(R-W) / 2$. Thus, $W$ (respectively $\left.R\right)$ is constant along the 1-characteristic curves (respectively the 4-characteristic curves). What is more, it is a 1-strong Riemann invariant (respectively a 4strong Riemann invariant) meaning that it is constant through the 2,3 and 4 waves (respectively the 1 and 2,3 waves). In Figure 1 the domains of invariance of $\mathrm{R}$ and $\mathrm{W}$ are drawn.

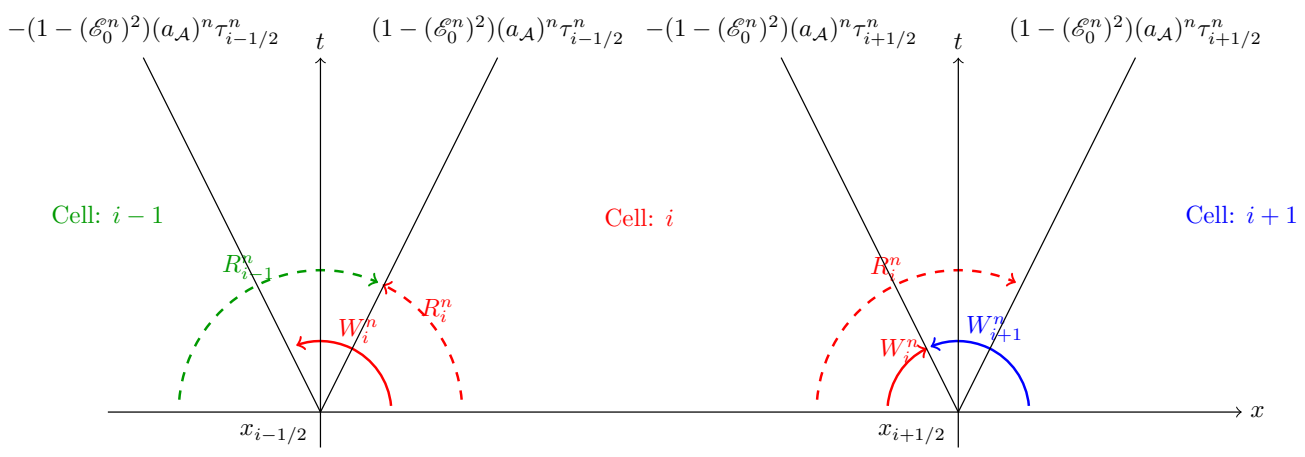

Figure 1: Strong Riemann Invariants Behaviors

As already noticed in [18, 29], if one formally removes the relaxation terms $\pm(p-\Pi) /\left(a_{\mathcal{A}} \mu\right)$ from the PDEs (3.14), the dynamics of $W$ and $R$ become totally uncoupled. Besides the energy flux depends only on these two quantities since according to equation (3.13):

$$
\Pi u=\frac{a_{\mathcal{A}}}{4}\left(R^{2}-W^{2}\right) .
$$

In the next subsection, the time-implicit scheme for the subsystem $\mathcal{A}$ is derived. It is based on the discretization of the simple transport dynamics of the quantities $W$ and $R$.

\section{The Acoustic Time-Implicit Scheme}

This section focuses on the space and time discretization of the acoustic subsystem $\mathcal{A}$. It is split in three parts. Subsections 4.1 and 4.2 provide the way to derive the time-implicit acoustic flux. Subsection 4.3 points out some properties of the overall IMEX scheme while subsection 4.4 concentrates of the discrete time-step construction.

\subsection{A One-dimensional Implicit-Solver for the Evolution Step}

The numerical resolution of the acoustic relaxation subsystem $\mathcal{A}^{\mu}$ is split into two steps: the first one, sometimes called the evolution step corresponds to the resolution of the homogeneous subsystem where the term $(p-\Pi) / \mu$ has been removed. It becomes active afterwards in an additional step which consists in solving:

$$
\partial_{t} \Pi=(p-\Pi) / \mu \text {. }
$$


However, in the present approach, the relaxation pressure $\Pi$ is relaxed instantaneously. Then, $\mu=0$ and the resolution of Eq 4.16 is replaced by the projection onto the equilibrium manifold:

$$
\left\{\mathbf{W}=[\rho, \rho u, \rho \Pi, \rho e]^{T}, \varepsilon=e-u^{2} / 2 \text {, s.t. } p(\rho, \varepsilon)=\Pi\right\} .
$$

98 For the sake of simplicity, this second step is called the projection step.

Suppose that the computational domain $\Omega=[0, L]$ is made of $N_{\text {cells }}$ cells. Let us define $\Delta x=L / N_{\text {cells }}$ (respectively $\Delta t$ ) the space-step (respectively the time-step) of the scheme. For $i \in\left[1, . ., N_{\text {cells }}\right]$ let us set $x_{i}=(i-1 / 2) \Delta x$, the coordinate of the cell center $i$ and $x_{i+1 / 2}=x_{i}+\Delta x / 2$, the coordinate of face $i+1 / 2$. Finally define $\left.\Omega_{i}=\right] x_{i-1 / 2}, x_{i+1 / 2}[$. Suppose that at time $t^{n+}$, the set of states $\mathbf{U}_{i}^{n+}=\left[\rho_{i}^{n+},(\rho u)_{i}^{n+},(\rho e)_{i}^{n+}\right]^{T}, i \in\left[1, . ., N_{\text {cells }}\right]$ produced after the resolution of the convective subsystem $C$ is provided to $\mathcal{A}^{\mu}$ as an initial piece-wise constant datum on $\sqcup_{i=1}^{N_{\text {cells }}} \Omega_{i}$. Then, by averaging the $W$-equation and the $R$-equation in 3.14 over $\left.\Omega_{i} \times\right] t^{n+}, t^{n}>t^{n+}$ [ one obtains:

$$
\begin{aligned}
& \frac{W_{i}^{\widetilde{n}}-W_{i}^{n+}}{\Delta t}-\frac{\left(1-\left(\mathscr{E}_{0}^{n}\right)^{2}\right)\left(a_{\mathcal{A}}\right)^{n+} \tau_{i}^{n+}}{\Delta x}\left(W_{i+1 / 2}\left(t^{n+}, t^{\widetilde{n}}\right)-W_{i-1 / 2}\left(t^{n+}, t^{\widetilde{n}}\right)\right)=0, \\
& \frac{R_{i}^{\widetilde{n}}-R_{i}^{n+}}{\Delta t}+\frac{\left(1-\left(\mathscr{E}_{0}^{n}\right)^{2}\right)\left(a_{\mathcal{A}}\right)^{n+} \tau_{i}^{n+}}{\Delta x}\left(R_{i+1 / 2}\left(t^{n+}, t^{\widetilde{n}}\right)-R_{i-1 / 2}\left(t^{n+}, \tilde{t}^{\widetilde{n}}\right)\right)=0,
\end{aligned}
$$

with $W_{i}^{n+}$ the spatial average over $\Omega_{i}$ at time $t^{n+}, W_{i+1 / 2}\left(t^{n+}, t^{\tilde{n}}\right)=\left(1 /\left(t^{\tilde{n}}-t^{n+}\right)\right) \times \int_{t^{n+}}^{t^{\tilde{n}}} \mathrm{~W}\left(x_{i+1 / 2} / t\right) d t$, and $\left(a_{\mathcal{A}}\right)^{n+}=$ $K \max _{i \in\left[1, N_{\text {cells }}\right]}\left(\rho_{i}^{n+}\left(c_{\mathcal{A}}\right)_{i}^{n+}\right), K>1$, the discrete acoustic relaxation constant fulfilling inequality 3.11 throughout the whole computational domain. Eventually the Mach-sensitive discrete parameter, built using the initial states $\mathbf{U}_{i}^{n}, i \in$ $\left[1, \ldots, N_{\text {cells }}\right]$ before the convective subsystem resolution, is given by:

$$
\begin{gathered}
\mathscr{E}_{0}^{n}=\max \left(M_{\text {inf }}, \min \left(M_{\text {max }}^{n}, 1\right)\right), \\
\text { with: } M_{\text {max }}^{n}=\max _{i \in\left[1, N_{\text {cells }}\right]}\left(\frac{\left|u_{i}^{n}\right|}{c_{i}^{n}}\right) .
\end{gathered}
$$

Remark 4.1. Averaging over a non-conservative term:

One can notice that, even if the $R$ and W PDEs are non-conservative because of the $\tau \partial_{x}($.$) operator, the fact$ that $\partial_{t} \tau=0$ in the acoustic subsystem of the current splitting and the piecewise continuous structure of the computed solution at time $t^{n}$ allow to derive exactly relations (4.18). This, is a key point to make sure that the discrete acoustic relaxation subsystem is conservative which, for the present IMEX scheme, is a necessary condition to ensure that the overall fractional step approach is conservative (see Proposition 4.2 below). Finally, it has to be mentioned that, up to the Mach-sensitive parameter, similar equations have already been obtained in the framework of the LagrangeProjection methods where a mass variable $m$ such as $\partial_{m}=\tau \partial_{x}$ is at stake. See [18] 17] for more details.

The end of the scheme derivation follows naturally by remembering that $W$ (respectively $R$ ) is constant over the 2,3 and 4-waves (respectively the 1 and 2,3-waves). Indeed, the signs of the different eigenvalues drawn in Figure 1 suggest that $W_{i+1 / 2}\left(t^{n+}, t^{\widetilde{n}}\right)$ (respectively $R_{i+1 / 2}\left(t^{n+}, t^{\widetilde{n}}\right)$ ) can be approximated by $W_{i+1}^{\widetilde{n}}$ (respectively by $R_{i-1}^{\widetilde{n}}$ ). Finally the two discrete dynamics write:

$$
\begin{aligned}
& \frac{W_{i}^{\widetilde{n}}-W_{i}^{n+}}{\Delta t}-\frac{\left(1-\left(\mathscr{E}_{0}^{n}\right)^{2}\right)\left(a_{\mathcal{A}}\right)^{n+} \tau_{i}^{n+}}{\Delta x}\left(W_{i+1}^{\widetilde{n}}-W_{i}^{\widetilde{n}}\right)=0, \\
& \frac{R_{i}^{\widetilde{n}}-R_{i}^{n+}}{\Delta t}+\frac{\left(1-\left(\mathscr{E}_{0}^{n}\right)^{2}\right)\left(a_{\mathcal{A}}\right)^{n+} \tau_{i}^{n+}}{\Delta x}\left(R_{i}^{\widetilde{n}}-R_{i-1}^{\widetilde{n}}\right)=0 .
\end{aligned}
$$

Thus, $\left(W_{i}^{\widetilde{n}}\right)_{i \in\left[1, N_{\text {cells }}\right]}\left(\right.$ respectively $\left.\left(R_{i}^{\widetilde{n}}\right)_{i \in\left[1, N_{\text {cells }}\right]}\right)$ is solution of an uncoupled linear system involving an upper-bidiagonal matrix (respectively a lower-bidiagonal matrix). If transmissive boundary conditions are used by introducing fictitious states $\underline{\mathbf{U}}_{0}^{\widetilde{n}}=\underline{\mathbf{U}}_{1}^{\widetilde{n}}$ and $\underline{\mathbf{U}}_{N_{\text {cells }}+1}^{\widetilde{n}}=\underline{\mathbf{U}}_{N_{\text {cells }}}^{\widetilde{n}}$, then $W_{N_{\text {cells }}+1}^{\widetilde{n}}=W_{N_{\text {cells }}}^{\widetilde{n}}$ and $W_{0}^{\widetilde{n}}=W_{1}^{\widetilde{n}}$. The matrices involved in 4.20) are then non singular since all their diagonal terms are strictly positive. What is more their bidiagonal structure, inherited from the transport dynamics of the strong relaxation Riemann invariants $W$ and $R$, allows to invert them without using any particular linear solver. 


\subsection{Projection Step and Time-Implicit Acoustic Flux}

Once the two uncoupled linear systems $(4.20)$ have been solved, the time-implicit acoustic flux related to the subsystem $\mathcal{A}^{\mu}$ can be deduced immediately. It reads:

$$
\begin{aligned}
& \mathbf{H}_{\mathcal{A} i+1 / 2}^{\mu \widetilde{n}}=\left(1-\left(\mathscr{E}_{0}^{n}\right)^{2}\right)\left[\begin{array}{c}
0 \\
\left(\Pi_{\mathcal{P}}^{*}\right)_{i+1 / 2}^{\widetilde{n}} \\
\left(a_{\mathcal{A}}^{n+}\right)^{2}\left(u_{\mathcal{A}}^{*}\right)_{i+1 / 2}^{n} \\
\left(\Pi_{\mathcal{A}}^{*}\right)_{i+1 / 2}^{n}\left(u_{\mathcal{A}}^{*}\right)_{i+1 / 2}^{n}
\end{array}\right], \\
& \left(u_{\mathcal{A}}^{*}\right)_{i+1 / 2}^{\widetilde{n}}=\frac{R_{i}^{\widetilde{n}}+W_{i+1}^{\widetilde{n}}}{2}, \\
& \left(\Pi_{\mathcal{A}}^{*}\right)_{i+1 / 2}^{\widetilde{n}}=\frac{\left(a_{\mathcal{A}}\right)^{n+}\left(R_{i}^{\widetilde{n}}-W_{i+1}^{\widetilde{n}}\right)}{2} .
\end{aligned}
$$

In the present work, the projection step is performed instantaneously. Indeed, $\mu$ is forced to tend fictively towards zero such that $p=\Pi$. Particularly, the time-implicit acoustic pressure at face $i+1 / 2$ can be defined as: $\left(p_{\mathcal{A}}^{*}\right)_{i+1 / 2}^{n+1}=\left(\Pi_{\mathcal{A}}^{*}\right)_{i+1 / 2}^{n}$. The other flux quantities remain invariant through the projection step and one can rewrite $\left(u_{\mathcal{A}}^{*}\right)_{i+1 / 2}^{\tilde{n}}$ as $\left(u_{\mathcal{A}}^{*}\right)_{i+1 / 2}^{n+1}$. Finally the time-implicit scheme for the acoustic subsystem $\mathcal{A}$ writes:

$$
\begin{aligned}
& \mathbf{U}_{i}^{n+1}=\mathbf{U}_{i}^{n+}-\frac{\Delta t}{\Delta x}\left(\mathbf{H}_{\mathcal{A}_{i+1 / 2}^{n+1}}-\mathbf{H}_{\mathcal{A}_{i-1 / 2}^{n+1}}\right), \\
& \text { with } \mathbf{H}_{\mathcal{H}_{i+1 / 2}^{n+1}}^{n+1}=\left(1-\left(\mathscr{E}_{0}^{n}\right)^{2}\right)\left[\begin{array}{c}
0 \\
\left(p_{\mathcal{H}}^{*}\right)_{i+1 / 2}^{n+1 / 2} \\
\left(p_{\mathcal{A}}^{*}\right)_{i+1 / 2}^{n+1}\left(u_{\mathcal{A}}^{*}\right)_{i+1 / 2}^{n+1}
\end{array}\right] .
\end{aligned}
$$

One can notice that, up to the factor $\left(1-\left(\mathscr{E}_{0}\right)^{2}\right)$, the obtained time-implicit acoustic flux is identical to the one derived in [17, 29]. Furthermore, the relaxation constant $a_{\mathcal{A}}$ makes it independent of the fluid EOS. As already stated, it requires no particular linear solver since the two uncoupled systems 4.20 can be inverted by hand. Besides, as proved in Appendix A, solving the discrete momentum equation of 4.22 is equivalent to setting:

$$
u_{i}^{n+1}=\frac{R_{i}^{n+1}+W_{i}^{n+1}}{2},
$$

provided that $u_{i}^{n+}=\frac{R_{i}^{n+}+W_{i}^{n+}}{2}$.

In the sequel, some additional properties of the overall IMEX fractional step are presented.

\subsection{The IMEX Scheme Properties}

Three properties are underlined below. The two first deal with the conservativity and the maximum principle whereas the last one concerns the discrete preservation of the Riemann Invariants of single contact discontinuities.

Proposition 4.2. Conservativity and maximum principle of the overall IMEX scheme:

- Conservativity:

Let us formally introduce $\mathbf{H}_{C}{ }_{i+1 / 2}^{n}\left(\mathbf{U}_{i}^{n}, \mathbf{U}_{i+1}^{n}\right)$ the time-explicit numerical flux associated to the subsystem $C$ (see [19. 20] or (A.7] in Appendix A for a definition). Then, the overall IMEX scheme is conservative and writes:

$$
\mathbf{U}_{i}^{n+1}=\mathbf{U}_{i}^{n}-\frac{\Delta t}{\Delta x}\left(\mathbf{H}_{C i+1 / 2}^{n}\left(\mathbf{U}_{i}^{n}, \mathbf{U}_{i+1}^{n}\right)+\mathbf{H}_{\mathcal{H}_{i+1 / 2}^{n+1}}^{n+\mathbf{H}_{C i-1 / 2}^{n}}\left(\mathbf{U}_{i-1}^{n}, \mathbf{U}_{i}^{n}\right)-\mathbf{H}_{\mathcal{A}_{i-1 / 2}^{n+1}}^{n+1}\right) .
$$

- Maximum principle:

Consider $\rho \phi$ a given conservative variable such as $\forall(x, t): \partial_{t}(\rho \phi)+\partial_{x}(\rho \phi u)=0 . \quad$ Assume that $(\rho \phi)_{i}^{n} \in$ $\left[(\rho \phi)_{\text {Min }},(\rho \phi)_{\text {Max }}\right]$. Then, the maximum principle preservation $(\rho \phi)_{i}^{n+1} \in\left[(\rho \phi)_{\text {Min }},(\rho \phi)_{\text {Max }}\right]$ depends only on the convective sub-step discretization. It naturally holds under a non-restrictive sufficient condition written in [19]: p.17, Lemma 1 (Positivity of intermediate density). 
It is well known that the above global conservativity result, which in this case is directly obtained because the acoustic sub-step discretization is conservative by construction, is a necessary step in order to capture the Euler physical shock fronts (see [30]). As for the maximum principle preservation for purely convected quantities, it can be considered as a first step towards the $L^{\infty}$ stability of the overall scheme.

Proposition 4.3. Discrete preservation of the contact discontinuity Riemann invariants:

Assume that the equation of state is such that $(\rho \varepsilon)^{E O S}(\rho, p)=C(p) \rho+B(p)$, with $p \rightarrow C(p)$ and $p \rightarrow B(p)$ smooth functions such as $(\rho \varepsilon)_{\mid \rho}^{E O S}: p \rightarrow C(p) \rho+B(p)$ is injective on the domain of definition of $p$ (see [31] or Appendix A] for an explanation of such hypothesis); then, the overall IMEX scheme exactly preserves the constant velocity and the constant pressure of an isolated contact discontinuity from one time-step to another.

According to [31], the above general expression of $(\rho \varepsilon)^{E O S}(\rho, p)$ belongs to the category of the "T1" equation of state. One can notably notice that the stiffened gas EOS: $\rho \varepsilon=p+\gamma \Pi$, is included in it. However the Van der Waals EOS: $\rho \varepsilon=\frac{\left(p+a \rho^{2}\right)(1-b \rho)}{\gamma-1}-a \rho^{2}$ is out of it. The above proposition will be useful in the sequel to detect the appearance of instabilities related to high convective Courant number $\mathscr{C}_{|u|}$. Proofs, including a brief description of the time-explicit convective flux related to subsystem $C$, can be found in Appendix A. The next subsection is devoted to the discrete time-step specification.

\subsection{Construction of the numerical time-step}

The time-step of the IMEX scheme is built using the convective eigenvalues $\lambda_{1}^{C}$ and $\lambda_{3}^{C}$ written in equation 2.5. As described in [19, 20], the convective subsystem $C$ is discretized using the same relaxation techniques as the one described in Section 3 The eigenvalues of the resulting relaxation system $C^{\mu}$ then write: $\lambda_{1}^{C, \mu}=u-\mathscr{E}_{0} a_{C} \tau, \lambda_{2,3}^{C, \mu}=u$ and $\lambda_{4}^{C, \mu}=u+\mathscr{E}_{0} a_{C} \tau$. They are related to the subcharacteristic condition $a_{C}>\rho c_{C}$.

For a given convective Courant number $\mathscr{C}_{C}$, the time-step at the $n$-th iteration of the numerical scheme is:

$$
\begin{aligned}
& \Delta t_{C}^{n}=\mathscr{C}_{C} \frac{\Delta x}{\max _{i+1 / 2}\left(\max \left(\left|u_{i}^{n}-\mathscr{E}_{0}^{n}\left(a_{C}\right)_{i+1 / 2}^{n} \tau_{i}^{n}\right|,\left|u_{i+1}^{n}+\mathscr{E}_{0}^{n}\left(a_{C}\right)_{i+1 / 2}^{n} \tau_{i+1}^{n}\right|\right)\right)}, \\
& \left(a_{C}^{n}\right)_{i+1 / 2}=K \max \left(\rho_{i}^{n}\left(c_{C}\right)_{i}^{n}, \rho_{i+1}^{n}\left(c_{C}\right)_{i+1}^{n}\right), K>1 .
\end{aligned}
$$

Let us assume that the stiffness of the discrete pressure gradient in the acoustic subsystem $\mathcal{A}$ has been completely removed thanks to the time-implicit integration. In that case one should expect that the present IMEX scheme is stable under the convective CFL condition: $\mathscr{C}_{C} \approx 1$.

From a numerical stability point of view, the time-step definition $(4.25)$ is thus the only admissible one regarding the waves produced by the convective subsystem. However, these waves never exist in the overall Euler system. For low Mach number flows under convective time-scales, the acoustic waves have vanished and the leading phenomenon is driven by the material velocity $u$. For that reason, one can introduce another time-step as:

$$
\Delta t^{n}=\mathscr{C}_{|u|} \frac{\Delta x}{\max _{i}\left(\left|u_{i}^{n}\right|\right)} .
$$

Once again, let us stress that the time-step definition 4.26 is essentially motivated by physical considerations. In the context of IMEX schemes based on convective-acoustic splittings, its formulation has also the advantage of being completely independent of the way to split as well as the way to discretize the associated subsystems. That is why, in the following numerical results section, the cases appealing to a fine accuracy or stability comparison between IMEX schemes are performed using the physical and universal time-step formula 4.26 . On the contrary, more complicated configurations requiring the proposed approach to be stable in order to capture some specific asymptotic regimes at low Mach number are tested with the convective-like time-step 4.25. 


\section{Numerical Results}

The following section is devoted to one-dimensional numerical results produced by the present IMEX approach. Subsection 5.1 focuses on the sensitivity w.r.t $\mathscr{C}_{|u|}$ of the acoustic and material waves propagation. Subsection 5.2 mainly concentrates on grid-convergence studies completed by efficiency comparisons between time-explicit and time-implicit solvers. For the reasons given in the above paragraph, IMEX schemes involved in subsection 5.1 and 5.2 use the discrete time-step (4.26). Nevertheless for each value of $\mathscr{C}_{|u|}$ an estimation of $\mathscr{C}_{C}$ is systematically provided. Subsection 5.3 deals with the appearance, in a fluid endowed with a stiff equation of state, of strong pressure jumps in an uniformly low-Mach number flow. Finally, subsection 5.4 aims at testing the ability of the present approach to treat a larger application spectrum like weakly compressible flows. In the last two subsections, the proposed method is systematically combined with the convective-like time-step (4.25).

\subsection{Low Mach Isolated Waves}

The first part of the numerical results is dedicated to the influence of the Courant number on quantities varying through the acoustic or material waves. Indeed, it is well known (see [32, 33, 34, 35, 36, 37]) that full time-implicit schemes, even with the use of high-order accurate discretizations in space, are only accurate on $\sigma$-like waves when the time-steps $\Delta t$ are such that $\mathscr{C}_{\sigma}=(|\sigma| \Delta t) / \Delta x \approx 1$. In the following, one seeks to investigate the accuracy of the above IMEX fractional step with respect to $\mathscr{C}_{|u|}$ and compare it with an other IMEX Lagrange-Projection (LP) fractional step described in [17, 38] in the context of low Mach number flows. Two low Mach number cases are thus evaluated. The first one details the propagation of a single 3-shock wave while the second one corresponds to the evolution of a single contact wave. In both cases, a particular attention will be paid to the diffusive or stiffening effects associated with the increase of $\mathscr{C}_{|u|}$. Transmissive conditions are used at the inlet and the outlet of the computational domain.

\subsubsection{Isolated Shock Wave}

For this test case, the fluid is endowed with an ideal gas EOS:

$$
\varepsilon=\frac{p}{(\gamma-1) \rho}, \text { and } c=\sqrt{\frac{\gamma p}{\rho}},
$$

with $\gamma=7 / 5$, the heat capacity ratio. The left state of the considered 3-isolated shock wave is completely defined by:

$$
\rho_{L}^{0}=1\left(k g \cdot m^{-3}\right), p_{L}^{0}=10^{4}(P a), \text { and } u_{L}^{0}=M_{0} \times c_{L}^{0},
$$

with $M_{0}=10^{-2}$ the maximal Mach number of the flow. What is more, the shock wave speed $\sigma$ is fixed equal to $c_{L}^{0} \approx 118.32\left(\mathrm{~m} . \mathrm{s}^{-1}\right)$. The three remaining unknowns $\rho_{R}^{0}, u_{R}^{0}$ and $p_{R}^{0}$ are the solutions of the corresponding RankineHugoniot problem and can then be found analytically. Besides, the resulting right state abides by the Lax entropy criterion: $u_{R}^{0}+c_{R}^{0}<\sigma<u_{L}^{0}+c_{L}^{0}$. The analytical solution is then composed of a single 3-shock wave.

In Figure 2, the isolated 3-shock wave pressure profile is shown. The physical time of the simulation is such that the initial discontinuity located at $x_{0}=0.5 \mathrm{~m}$ stops at $x=0.75 \mathrm{~m}$. The mesh is made of $10^{3}$ cells.

Different curves are plotted; Sp- $(M)$ stands for the current splitting presented in Eqs (2.2) and (2.3) whereas Sp-LP refers to a Lagrange-Projection splitting method fully described in [17] and taken as a benchmark in this work. Besides the abbreviation "Exp" indicates that the acoustic part of the Sp- $(M)$ splitting (respectively the Sp-LP splitting) has been discretized using a time-explicit scheme detailed in [19, 20] (respectively [17]). In this case, the CFL condition is such that $\mathscr{C}_{E}=1$. On the contrary "Imp" refers to the above time-implicit approach.

Two different convective Courant numbers values have been tested in the implicit-explicit approaches: the first one $\mathscr{C}_{|u|}=0.01\left(\mathscr{C}_{C} \approx 2.37 \times 10^{-2}\right)$ has been deliberately chosen to provide time-steps close to those based on the constraint $\mathscr{C}_{E} \approx 1$ since formally $\mathscr{C}_{|u|}=M /(1+M) \mathscr{C}_{E}$ and $M=10^{-2}$. The other value $\mathscr{C}_{|u|}=0.3\left(\mathscr{C}_{C} \approx 7.11 \times 10^{-1}\right)$ corresponds to $\mathscr{C}_{E} \approx 30$ and is thus expected to be too high for the IMEX scheme to accurately follow the shock wave front.

In this low Mach number case, it turns out that the sharper pressure profiles are those provided by the time-explicit schemes complying with the constraint: $\mathscr{C}_{E} \approx 1$. On the contrary the higher is $\mathscr{C}_{E}$, the more diffused the shock profile is. Besides, for a fixed Euler Courant number $\mathscr{C}_{E} \approx 1$, the averaging effect of the time-implicit schemes relatively to the time-explicit ones can be observed as the profile of "Sp- $(M)$-Imp: $\mathscr{C}_{|u|}=0.01$ " is largely more diffused than "Sp-(M)-Exp: $\mathscr{C}_{E}=1 "$. 


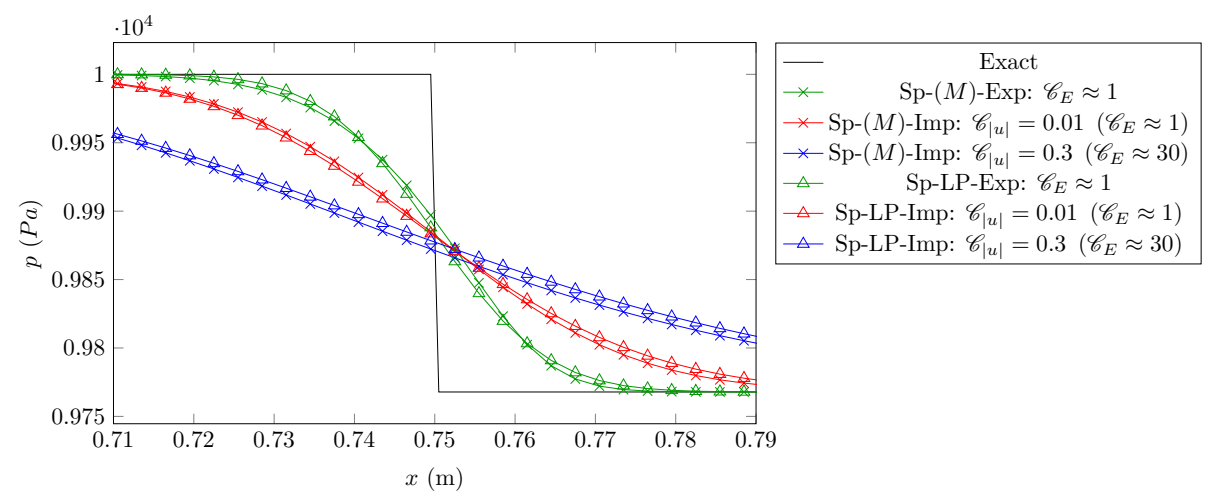

Figure 2: Pressure profile, Ideal Gas, $M=10^{-2}$, with $N_{\text {cells }}=10^{3}$

Finally, one can notice that, for all the Courant number values involved here, the $\mathrm{Sp}-(M)$ splitting is as accurate as the Sp-LP method. These results, obtained in the context of the Euler system, seem to be close to the predictions given by the linear stability analysis in [37].

The above simple test case, involving a fast acoustic wave related to a genuinely non-linear field, has allowed to test the robustness with respect to $\mathscr{C}_{|u|}$ of the present approach compared with the LP method. In the sequel the same study is performed on a slow material wave associated with a linearly degenerate field.

\subsubsection{Isolated Contact Discontinuity}

For the same thermodynamical law, a single contact discontinuity is created by imposing:

$$
\begin{aligned}
& \rho_{L}^{0}=1\left(\mathrm{~kg} \cdot \mathrm{m}^{-3}\right), \rho_{R}^{0}=0.125\left(\mathrm{~kg} \cdot \mathrm{m}^{-3}\right), \\
& p_{L}^{0}=p_{R}^{0}=p^{0}=10^{4}(P a), \\
& u_{L}^{0}=u_{R}^{0}=M_{0} \times c_{R}^{0}=u^{0},
\end{aligned}
$$

with $M_{0}=10^{-2}$. This wave linked to a linearly degenerate field propagates at speed $u_{0} \approx 3.35\left(\mathrm{~m} . \mathrm{s}^{-1}\right)$.

In Figure 3, one can observe the density profiles. As it was expected, the isolated contact discontinuity sharpens as the convective Courant number $\mathscr{C}_{|u|}$ reaches 1 . Once again, for the same Courant number, the profiles between the present IMEX scheme and the IMEX-LP scheme overlap quasi-perfectly.

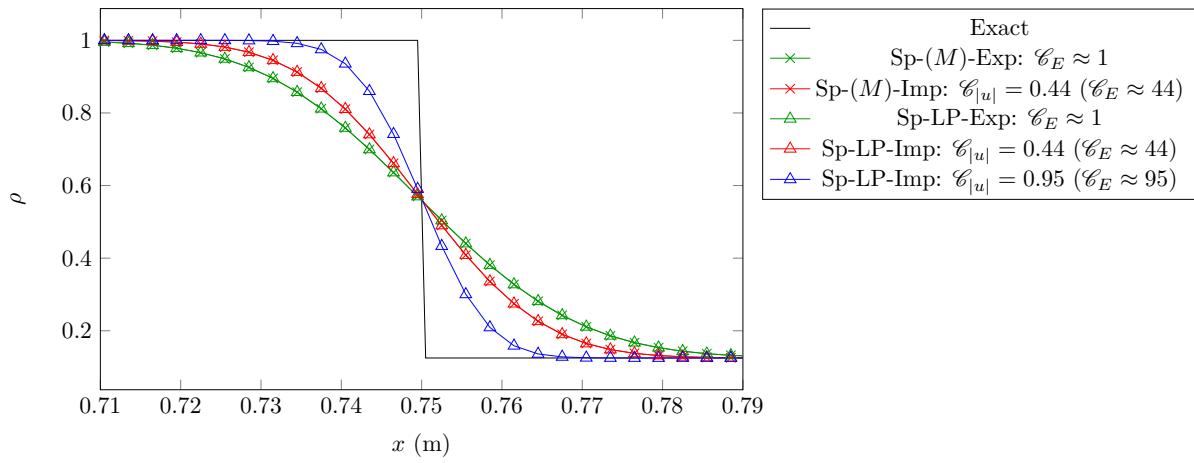

Figure 3: Density profile, Ideal Gas, $M_{\min }=10^{-2}$, with $N_{\text {cells }}=10^{3}$

However, one can notice that the ideal target associated to $\mathscr{C}_{|u|}=0.95 \approx 1$ has only been achieved for the LP method. Indeed, for this test case, the present splitting and the resulting IMEX approach triggers instabilities for $\mathscr{C}_{|u|}$ above 0.44. Trying to connect this threshold with a reconstructed value of the convective Courant number $\mathscr{C}_{C}$, one can 
define:

$$
\left(\mathscr{C}_{C}\right)_{i+1 / 2}^{n}=\frac{\max \left(\left|u_{i}^{n}-\mathscr{E}_{0}^{n}\left(a_{C}\right)_{i+1 / 2}^{n} \tau_{i}^{n}\right|,\left|u_{i+1}^{n}+\mathscr{E}_{0}^{n}\left(a_{C}\right)_{i+1 / 2}^{n} \tau_{i+1}^{n}\right|\right) \Delta t^{n}}{\Delta x},
$$

with $\Delta t^{n}$ the discrete time-step defined in equation (4.26) and used in this test case. In Figure 4 the value of $\left(\mathscr{C}_{C}\right)_{i+1 / 2}^{n}$ is displayed at the final simulation time and overall the computational domain. One can observe that the reconstructed

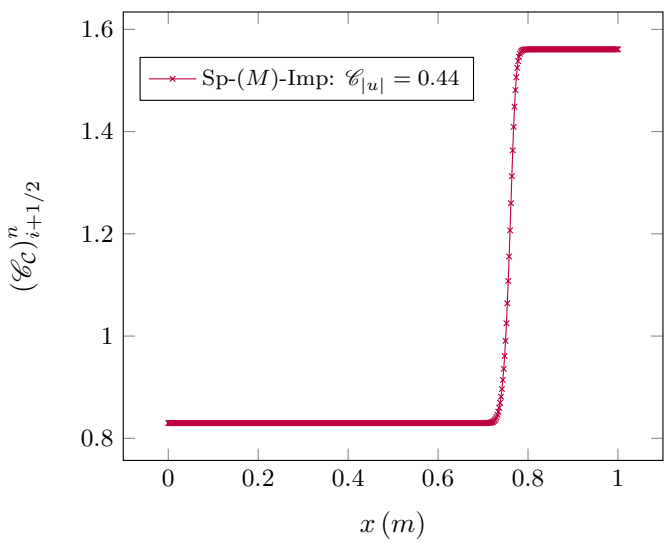

Figure 4: Reconstructed local convective Courant number

convective Courant number is strictly higher than one in the region where the Mach number is minimal. Let us recall that, as the term $\mathscr{E}_{0}$ decreases, the proportion of the pressure gradient that is discretized using a time-implicit integration increases as $\left(1-\mathscr{E}_{0}^{2}\right)$ according to the definition of the time-implicit acoustic flux 4.22 . Then, when $\mathscr{E}_{0}$ is small enough, the amount of numerical diffusion produced by the time-implicit part of the scheme is sufficient to balance the anti-diffusive effect brought by the time-explicit discretization if $\mathscr{C}_{C}>1$. Such a phenomenon is analyzed in [14, 15] using the modified equation tool.

Conversely, when $M_{0}$ is high enough, the splitting parameter $\mathscr{E}_{0}$ tends towards one so that the time-implicit numerical diffusion is canceled out. The classical time-explicit CFL condition $\mathscr{C}_{C} \approx 1$ is then retrieved.

Details dealing with the stability of the proposed IMEX scheme when applied to this test case with varying Mach number $M_{0}$ are provided in Appendix B The residual term $\mathscr{E}_{0} c_{C}$ prevents from reaching the material CFL condition $\mathscr{C}_{|u|}=1$ proved by Zakerzadeh in [16] for the IMEX LP scheme. This is the price to be paid after having introduced $\mathscr{E}_{0}^{2} p$ into the convective subsystem $C$. Nevertheless, in this test case, the stability of the proposed IMEX scheme holds under $\mathscr{C}_{C} \approx 1$ for any value of the flow Mach number. These numerical observations support a "Mach-uniform" $\mathscr{C}_{C}$-stability property of the present method.

In the sequel, accuracy and efficiency of the above IMEX schemes are compared with their full time-explicit versions in the context of low Mach number flows.

\subsection{A Low Mach Sod Shock Tube}

By imposing a common Courant number relying on the material velocity $u$, the above isolated wave test cases have pointed out some stability differences between the proposed IMEX scheme and the LP-IMEX method. In the sequel, one wishes to refine the comparison by adding some grid-convergence and efficiency results obtained from a multiple wave test case.

Herein, a low Mach number shock tube is computed. The fluid is endowed with the same previous ideal gas EOS. The initial conditions are made of a density discontinuity, a constant velocity, and a slightly discontinuous pressure:

$$
\begin{aligned}
& \rho_{L}^{0}=1 \mathrm{kgm}^{-3}, \rho_{L}^{0}=0.125 \mathrm{kgm}^{-3} \\
& u_{L}^{0}=u_{R}^{0}=u^{0}=1 \mathrm{~ms}^{-1} \\
& p_{L}^{0}=10080 \mathrm{~Pa}, p_{R}^{0}=10000 \mathrm{~Pa} .
\end{aligned}
$$


It results in a left-going 1-rarefaction wave, a 2-contact discontinuity propagating to the right and a right-going 3-shock wave. The maximal Mach number of the flow, reached in the head of the rarefaction wave, is equal to $1.26 \times 10^{-2}$.

Various time-explicit schemes have been tested: "no-Sp" corresponds to the case where $\mathscr{E}_{0}=1$ is imposed along the simulation. Thus, the splitting is not triggered. "Sp- $(\sqrt{M})$ " is the weighted splitting approach with $\mathscr{E}_{0}=$ $\max \left(\sqrt{M_{\text {inf }}}, \min \left(\sqrt{M_{\text {max }}^{n}}, 1\right)\right)$ while "Sp- $(M)$ " involves $\mathscr{E}_{0}$ defined in formula 4.19 which is a priori optimal for a time-explicit scheme, because, as shown in [19, 20], it minimizes the numerical diffusion of the subsystem $C$ in the low-Mach number case. Lastly, "Sp-LP" is again the Lagrange Projection splitting method, described in [17]. Besides, the mention "-corr" means that a low-Mach correction inspired from [39] and written in [19, 20] is triggered. As observed in [19, 20, 17, 38], it aims at considerably reducing the numerical diffusion in the case of low Mach number flows.

Regarding the time-implicit schemes, two values for $\mathscr{C}_{|u|}$ have been tested. As shown in Appendix E the ratio between $\mathscr{C}_{|u|}$ the convective Courant number based on $u^{0}$ and $\mathscr{C}_{E}^{0, *}$ the most constraining Euler Courant number is:

$$
\mathscr{C}_{|u|}^{u^{0}} \approx 1.5 \times 10^{-3} \mathscr{C}_{E}^{0, *}
$$

Then, the selected convective Courant numbers are $\mathscr{C}_{|u|}=1.5 \times 10^{-2}\left(\mathscr{C}_{E}^{0, *} \approx 10, \mathscr{C}_{C} \approx 6.34 \times 10^{-2}\right)$ and $\mathscr{C}_{|u|}=4.5 \times 10^{-2}$ $\left(\mathscr{C}_{E}^{0, *} \approx 30, \mathscr{C}_{C} \approx 1.9 \times 10^{-1}\right)$.
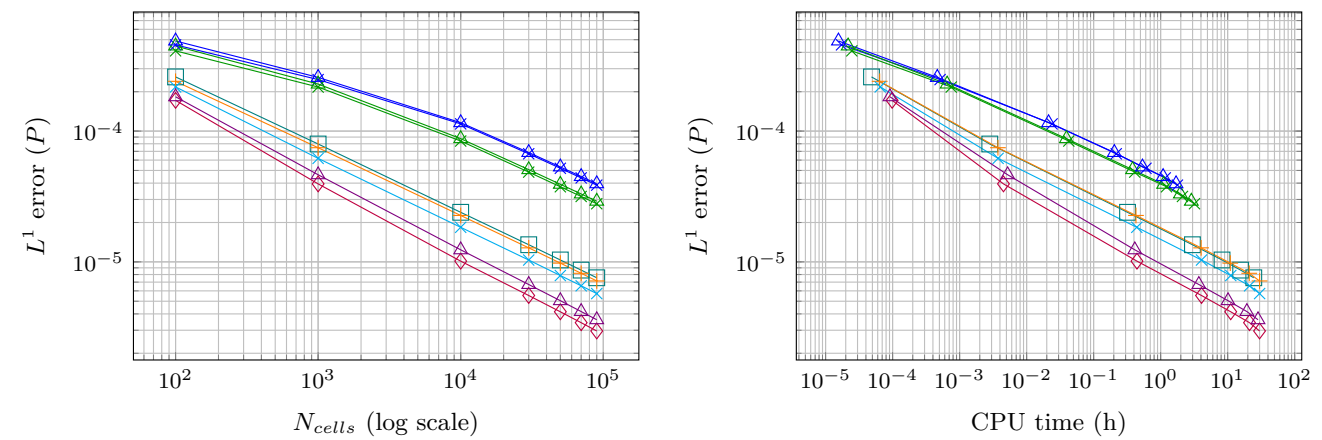

\begin{tabular}{|c|c|c|c|c|c|}
\hline$\square$ & No-Sp & + & $\mathrm{Sp}-(\sqrt{M})$ & $\diamond$ & $\mathrm{Sp}-(\sqrt{M})$-corr \\
\hline$\rightarrow$ & Sp- $(M)$ & $*$ & Sp-(M)-Imp- $\mathscr{C}_{E} 10$ & $\rightarrow$ & Sp- $(M)-\operatorname{Imp}-\mathscr{C}_{E} 30$ \\
\hline$\triangle$ & Sp-LP-corr & $\triangle$ & Sp-LP-Imp- $\mathscr{C}_{E} 10$ & $\triangle$ & Sp-LP-Imp- $\mathscr{C}_{E} 30$ \\
\hline
\end{tabular}

Figure 5: Convergence curve (left), Efficiency curve (right) for the pressure variable $p: M=1.26 \times 10^{-2}$
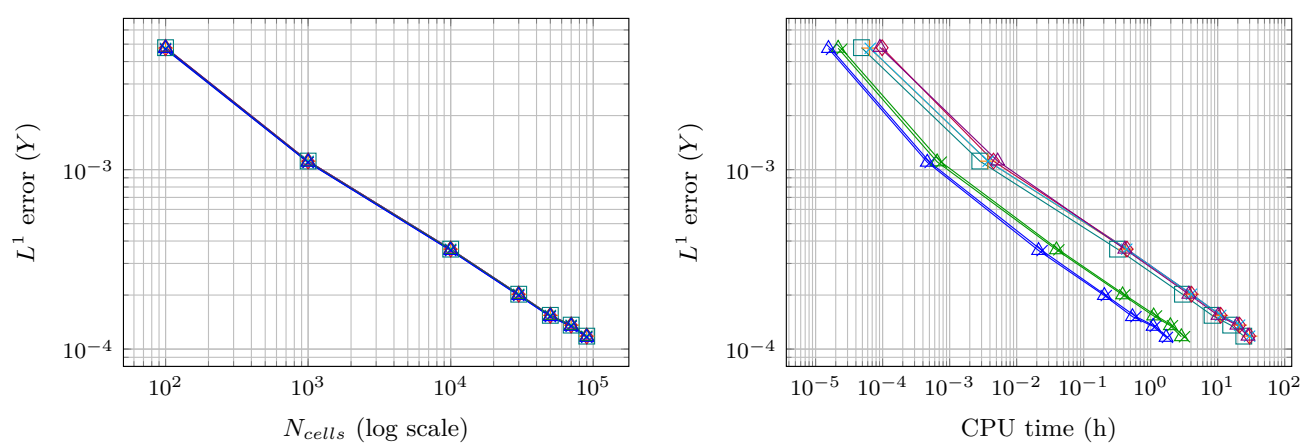

\begin{tabular}{|cccccc|}
$\square$ & No-Sp & - & Sp- $(\sqrt{M})$ & $\smile$ & Sp- $(\sqrt{M})$-corr \\
$\rtimes$ & Sp- $(M)$ & $\star$ & Sp- $(M)-I m p-\mathscr{C}_{E} 10$ & $\star$ & Sp- $(M)-I m p-\mathscr{C}_{E} 30$ \\
$\triangle$ & Sp-LP-corr & $\triangle$ & Sp-LP-Imp- $\mathscr{C}_{E} 10$ & $\triangle$ & Sp-LP-Imp- $\mathscr{C}_{E} 30$ \\
\hline
\end{tabular}

Figure 6: Convergence curve (left), Efficiency curve (right) for the passive tracer variable $Y: M=1.26 \times 10^{-2}$ 
In this test case, in order to isolate the effect of the IMEX scheme on the contact discontinuity, a passive tracer $Y$ has been added to the Euler system. Its PDE writes:

$$
\partial_{t}(\rho Y)+\partial_{x}(\rho Y u)=0
$$

Starting from $Y_{L}^{0}=1$ and $Y_{R}^{0}=0.5$, the exact solution jumps only through the contact discontinuity.

Figure 5 (respectively Figure 6) presents the convergence curve and the efficiency curve for the pressure (respectively $Y$ ) variable. As already pointed out in [19, 20], for such a low Mach number, and focusing on the pressure (or any variable jumping through the genuinely non-linear fields), the time-explicit schemes with the low Mach number correction Sp- $(\sqrt{M})$-corr and Sp-LP-corr, are the most accurate as well as the most efficient. As already observed in the isolated 3-shock wave test case, time-implicit schemes such that $\mathscr{C}_{E}>1$ are less accurate than any of the timeexplicit schemes. Besides, according to the pressure efficiency curve, this lack of accuracy is not compensated by a substantial gain in CPU time. Indeed, for a given pressure $L^{1}$-error level, time-implicit schemes are still more CPU-consuming than the time-explicit ones.

In the case of the passive tracer $Y$, no specific difference on the convergence curve can be noticed between the timeexplicit and the time-implicit schemes. Indeed, the convective Courant number $\mathscr{C}_{|u|} \leq 4.5 \times 10^{-2}$ is still very far from one. Thus for every scheme, numerical diffusion has uniformly smoothed the variables only jumping through the slow material wave. Nevertheless, as shown in Table 1. time-implicit schemes are clearly more efficient, for the $Y$ variable, than the time-explicit ones, the latter being 7 (respectively 13) times more CPU-consuming than time-implicit schemes when setting $\mathscr{C}_{E}^{0, *}=10$ (respectively $\mathscr{C}_{E}^{0, *}=30$ ).

\begin{tabular}{|c|c|c|}
\hline Ncells & $\mathscr{C}_{E}=10$ & $\mathscr{C}_{E}=30$ \\
\hline $10^{2}$ & 1.97 & 2.80 \\
\hline $10^{3}$ & 3.75 & 5.51 \\
\hline $10^{4}$ & 7.52 & 13.36 \\
\hline $3 \times 10^{4}$ & 7.26 & 13.71 \\
\hline $5 \times 10^{4}$ & 6.74 & 13.66 \\
\hline $7 \times 10^{4}$ & 7.39 & 13.34 \\
\hline $9 \times 10^{4}$ & 7.67 & 13.78 \\
\hline
\end{tabular}

Table 1: $T_{\mathrm{No}-\mathrm{Sp}}^{\mathrm{CPU}} / T_{\mathrm{Sp}-(M) \text {-Imp }}^{\mathrm{CPU}}$

As announced in the introduction, one of the objective of the present approach is to capture high amplitude pressure jumps in low velocity areas as it is noticed in water-hammer events. This is the purpose of the next numerical example.

\subsection{A Double Riemann Problem With Stiff Thermodynamics}

In the following test case, two Riemann problems are triggered at $x_{0}=0.55 \mathrm{~m}$ and $x_{1}=1.23 \mathrm{~m}$ in a domain of length $L=2 m$. The three initial condition areas are written in Table 2 .

\begin{tabular}{|c|c|c|c|}
\hline & Left state $\left(x<x_{0}\right)$ & Middle state $\left(x_{0}<x<x_{1}\right)$ & Right state $\left(x_{1}<x\right)$ \\
\hline$\rho\left(k g . \mathrm{m}^{-3}\right)$ & $\rho_{L}^{0}=10^{3}$ & $\rho_{\mathrm{m}}^{0}=9.98 \times 10^{2}$ & $\rho_{R}^{0}=9.97 \times 10^{2}$ \\
\hline$u\left(m \cdot \mathrm{s}^{-1}\right)$ & $u_{L}^{0}=1$ & $u_{\mathrm{m}}^{0}=1$ & $u_{R}^{0}=1$ \\
\hline$p($ bar $)$ & $p_{L}^{0}=10^{3}$ & $p_{\mathrm{m}}^{0}=10$ & $p_{R}^{0}=1$ \\
\hline$Y$ & $Y_{L}^{0}=0.7$ & $Y_{\mathrm{m}}^{0}=0.2$ & $Y_{R}^{0}=0.1$ \\
\hline
\end{tabular}

Table 2: Double Riemann Problem: initial conditions

The fluid is endowed with a stiffened gas EOS, i.e.:

$$
\varepsilon=\frac{p+P_{\infty}}{(\gamma-1) \rho}, \text { and } c=\sqrt{\frac{\gamma\left(p+P_{\infty}\right)}{\rho}},
$$


with $\gamma=7.5$ and $P_{\infty}=3 \times 10^{3}$ bar. These constants have been chosen such that, at a given temperature $T=295 \mathrm{~K}$, the value of the speed of sound is approximately $c \approx 1.5 \times 10^{3} \mathrm{~m} \cdot \mathrm{s}^{-1}$. It is representative of the liquid water behavior.

The analytical solution of the present test case is composed of two successive shock tubes whose waves are similar to these presented in subsection 5.2. The maximal Mach number of the Riemann problem initially located at $x_{0}$ (respectively $x_{1}$ ) is $M_{\max }^{x_{0}} \approx 2 \times 10^{-2}$ (respectively $M_{\max }^{x_{1}} \approx 8.6 \times 10^{-4}$ ). Thus, the flow Mach number is uniformly low. Besides the important variations of the Mach number are associated with strong velocity fluctuations. It passes from $u \approx 32 \mathrm{~m} \cdot \mathrm{s}^{-1}$ in the contact wave related to the Riemann problem located at $x_{0}$ to $u \approx 1.3 \mathrm{~m} \cdot \mathrm{s}^{-1}$ in the contact wave of the one located at $x_{1}$. That is why in the sequel, the Riemann problem initially located at $x_{0}$ is referred as "high-velocity" shock tube, whereas the one initially located at $x_{1}$ is called "low-velocity" shock tube.

In any case, the stiffened gas EOS combined with high initial pressure discontinuities produce strong shock waves in both Riemann problems. The pressure jump amplitude associated with the high-velocity shock tube (respectively the low-velocity shock tube) is approximately 480 bar (respectively 4.5 bar). The above test case represents the sudden occurrence of high amplitude shock waves in a uniformly low Mach number flow as it can be observed in water-hammer scenarios.

In the sequel, a comparison between the IMEX fractional steps Sp- $(M)$-Imp and Sp-LP-Imp is done. For each numerical scheme, the time-step is only provided by the waves produced by the convective subsystem: $\Delta t_{C}^{n}$ written in formula $(4.25)$ for the present splitting and

$$
\begin{aligned}
& \Delta t_{\mathrm{LP}}^{n}=\mathscr{C}_{C} \frac{\Delta x}{\left.\max _{i+1 / 2}\left(\left(u_{\mathcal{P}}^{*}\right)_{i-1 / 2}^{n}\right)^{+}-\left(\left(u_{\mathcal{P}}^{*}\right)_{i+1 / 2}^{n}\right)^{-}\right)}, \\
& \left(u_{\mathcal{P}}^{*}\right)_{i+1 / 2}^{n}=\frac{u_{i+1}^{n}+u_{i}^{n}}{2}-\frac{1}{2 a_{i+1 / 2}^{n}}\left(p_{i+1}^{n}-p_{i}^{n}\right), \\
& a_{i+1 / 2}^{n}=K \max \left(\rho_{i}^{n} c_{i}^{n}, \rho_{i+1}^{n} c_{i+1}^{n}\right), K>1,
\end{aligned}
$$

for the Lagrange-Projection splitting (see [17]). For both time-steps, the convective Courant number $\mathscr{C}_{C}$ is set to 0.9 . What is more, the discrete splitting parameter $\mathscr{E}_{0}^{n}$ defined in Eqs (4.19) is here enriched for the present test case with a simple hand-made shock detector:

$$
\begin{aligned}
& \mathscr{E}_{0}^{n}=\max \left(M_{\text {inf }}, \min \left(\max \left(M_{\text {max }}^{n}, M_{S, \text { max }}^{n}\right) 1\right)\right), \\
& \text { with } \quad M_{\text {max }}^{n}=\max _{i \in\left[1, N_{\text {cells }}\right]}\left(\frac{\left|u_{i}^{n}\right|}{c_{i}^{n}}\right), \quad M_{S, \text { max }}^{n}=\max _{i \in\left[1, N_{\text {cells }}\right]}\left(\frac{\left|\left(\sigma_{S}\right)_{i+1 / 2}^{n}\right|}{\max \left(c_{i+1}^{n}, c_{i}^{n}\right)}\right),
\end{aligned}
$$

and

$$
\left(\sigma_{S}\right)_{i+1 / 2}^{n}=\left\{\begin{array}{cl}
\frac{(\rho u)_{i+1}^{n}-(\rho u)_{i}^{n}}{\rho_{i+1}^{n}-\rho_{i}^{n}} & \text { if }\left|\rho_{i+1}^{n}-\rho_{i}^{n}\right|>\epsilon^{\text {thres }} \max \left(\rho_{i+1}^{n}, \rho_{i}^{n}\right) \\
0 & \text { otherwise, }
\end{array}\right.
$$

where $\epsilon^{\text {thres }}=10^{-8}$. One can notice that in Eqs 5.36, $\left(\sigma_{S}\right)_{i+1 / 2}^{n}$ corresponds to the exact shock front speed formula in the case of an isolated shock wave separating the states $\mathbf{U}_{i}^{n}$ and $\mathbf{U}_{i+1}^{n}$. If one considers the waves related to the genuinely non-linear fields, i.e. $u \pm c$, the Lax entropy conditions related to admissible shock waves then give:

$$
u_{i}^{n} \pm c_{i}^{n}>\left(\sigma_{S}\right)_{i+1 / 2}^{n}>u_{i+1}^{n} \pm c_{i+1}^{n} .
$$

Hence, in the case of a low Mach number flow, for which $\left|u_{i+1}^{n}\right| / c_{i+1}^{n} \ll 1$ and $\left|u_{i}^{n}\right| / c_{i}^{n} \ll 1$, the term $\left|\left(\sigma_{S}\right)_{i+1 / 2}^{n}\right|$ should approximately belong to $] \min \left(c_{i+1}^{n}, c_{i}^{n}\right), \max \left(c_{i+1}^{n}, c_{i}^{n}\right)[$. Besides, the low compressibility of a fluid endowed with the above stiffened gas EOS involves very small variations of $\rho$ if $p \ll P_{\infty}$. Then, $c$ should remain constant at least in the low-velocity shock tube area. Thus, in the case of shock waves, $M_{S, \max }^{n}$ should be of order one and the present IMEX approach should turn into a fully time-explicit Godunov-like solver. 
The number of cells is $2 \times 10^{3}$ and the simulation time $T_{\text {end }}=1.95 \times 10^{-4} \mathrm{~s}$ is set in order to avoid interactions between the 3-right-going shock wave of the high-velocity shock tube and the 1-left-going rarefaction wave of the low-velocity shock tube. Transmissive boundary conditions have been used.

Figure 7 and Figure 8 show the pressure and the velocity profiles for both successive shock tubes. Figure 9 displays the passive tracer profile $Y$ which only jumps through the contact waves.

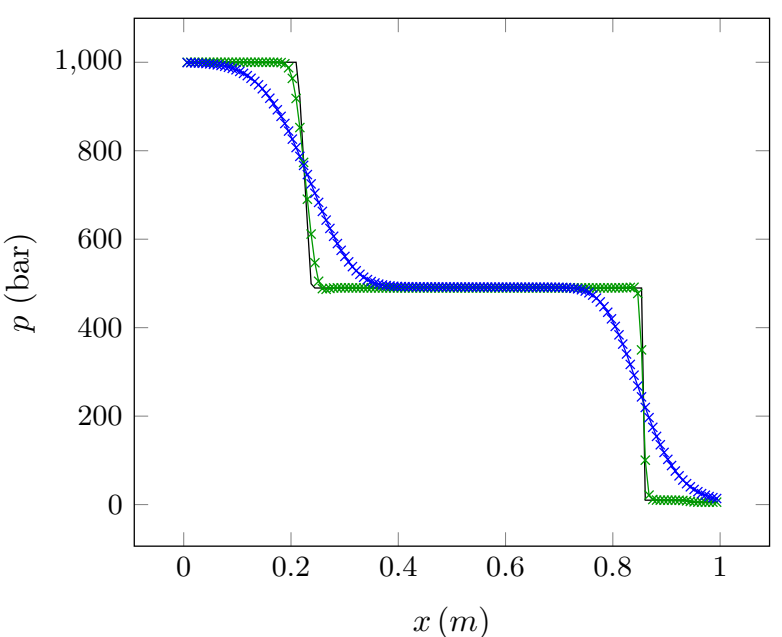

(a) Pressure profile: high velocity area

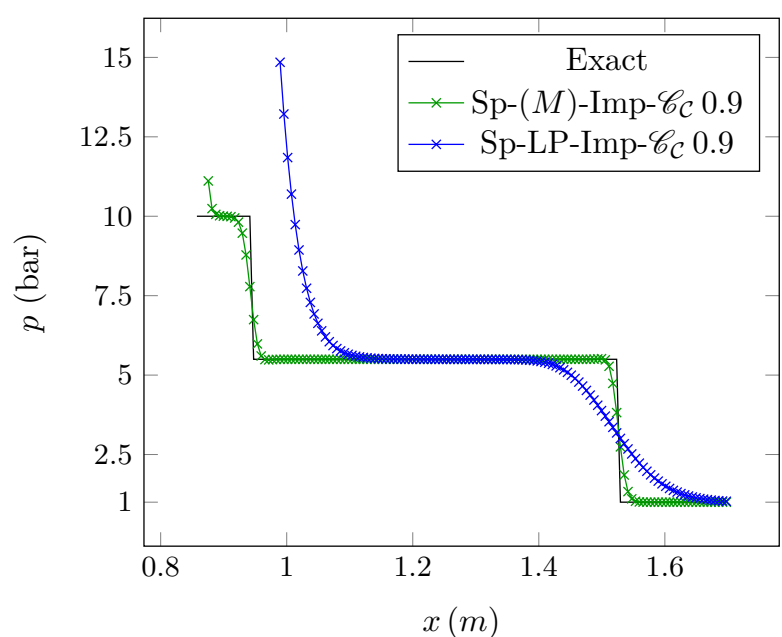

(b) Pressure profile: low velocity area

Figure 7: Pressure profile, stiffened gas EOS, $N_{\text {cells }}=2 \times 10^{3}$

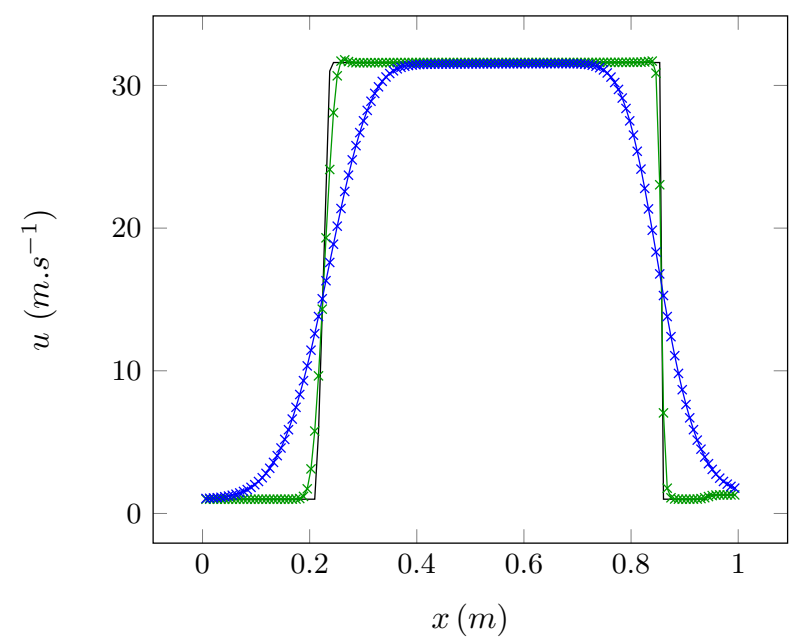

(a) Velocity profile: high velocity area

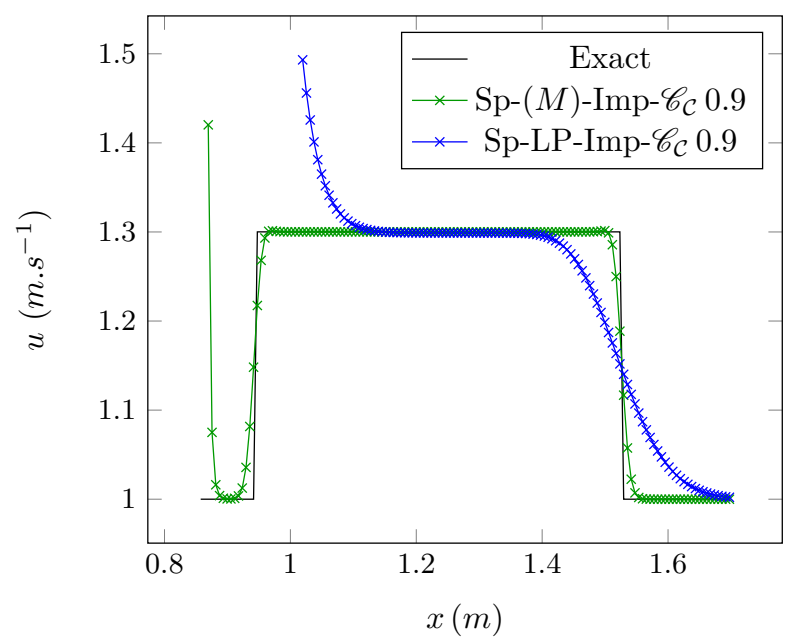

(b) Velocity profile: low velocity area

Figure 8: Velocity profile, stiffened gas EOS, $N_{\text {cells }}=2 \times 10^{3}$

In the case of Sp-(M)-Imp, once the different waves have appeared, the measured shock detector $M_{S, \text { max }}^{n}$ is of order one through the rarefaction and the shock waves. Then after several time-steps $\mathscr{E}_{0}^{n}=1$, and a full time-explicit 
Riemann solver associated with an Euler-like time-steps,

$$
\begin{aligned}
& \Delta t_{C}^{n} \approx \mathscr{C}_{C} \frac{\Delta x}{\max _{i+1 / 2}\left(\max \left(\left|u_{i}^{n}-c_{i}^{n}\right|,\left|u_{i+1}^{n}+c_{i+1}^{n}\right|\right)\right)}, \\
& \mathscr{C}_{C}=0.9,
\end{aligned}
$$

is recovered. Thanks to the degree of freedom offered by $\mathscr{E}_{0}$, the present fractional step is therefore able to select the appropriate time-discretization for the acoustic flux and in the same time the right time-step adapted to the physics of interest. On the contrary, the acoustic part of the Lagrange-Projection fractional step is still discretized using a time-implicit scheme linked to a CFL condition based on a very low material velocity such as $u \ll c$. Then, it is far less accurate at capturing stiff rarefaction and shock waves in both "high-" and "low-velocity" shock tubes. One can notably observe in Figure $7 \mathrm{~b}$ and Figure $8 \mathrm{~b}$ that, on this mesh, Sp-LP-Imp fails to capture the initial state $\left(u_{\mathrm{m}}^{0}, p_{\mathrm{m}}^{0}\right)$ between the 1-right-going shock wave of the high-velocity shock tube and the 3-left-going rarefaction wave of the low-velocity one. This is due to an excessive numerical dissipation through the associated waves.

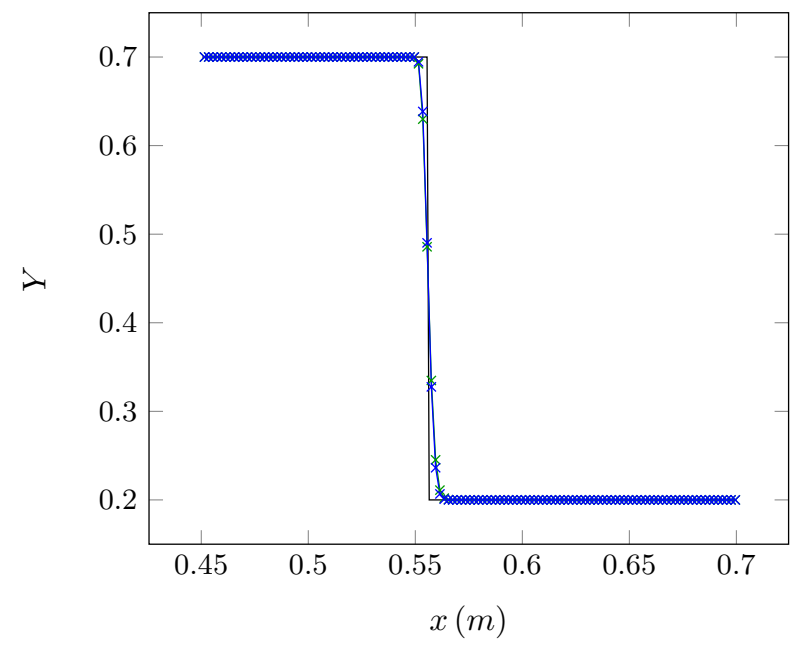

(a) $Y$ profile: high velocity area

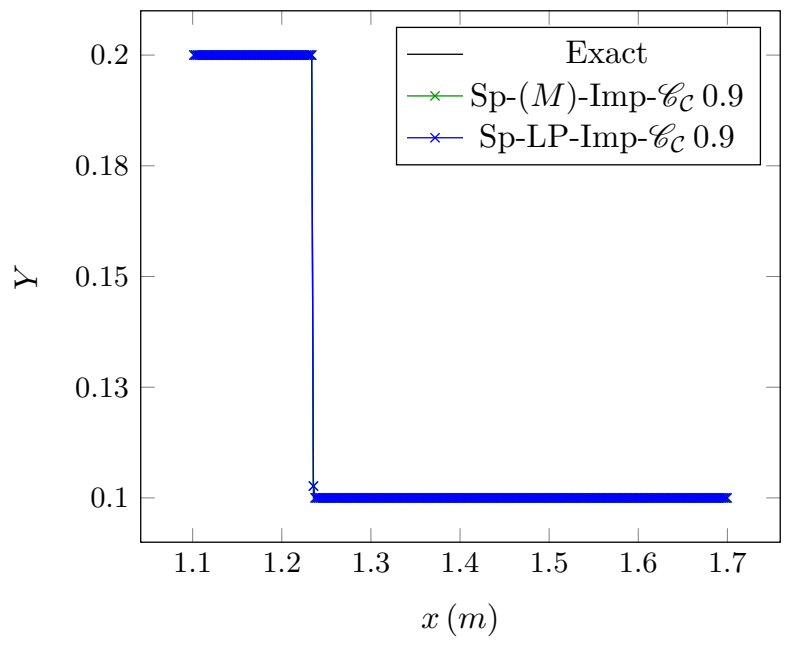

(b) $Y$ profile: low velocity area

Figure 9: $Y$ profile, stiffened gas EOS, $N_{\text {cells }}=2 \times 10^{3}$

Eventually, as seen in Figure 9, both IMEX schemes produce similar results regarding the accuracy of the $Y$ profile. It can be explained because the simulation time $T_{\text {end }}$ is based on the fast acoustic waves, the exact contact discontinuities only move a few space-steps. One can assume that, on a larger time of simulation, the Sp-LP-Imp should be more accurate on $Y$ than Sp- $(M)$-Imp. Indeed, the LP time-step is directly based on slow material velocities.

Therefore, this test case has shown that the degree of freedom offered by the splitting parameter $\mathscr{E}_{0}$ allows to automatically switch from a scheme designed to follow slow material dynamics to a scheme able to capture fast acoustic waves even when the flow Mach number is low.

The next section proposes to widen the application fields of the present IMEX scheme. Indeed, it aims at assessing the scheme ability to seize some simple constant states as well as weakly compressible flows.

\subsection{Applications to some other asymptotic regimes \\ 5.4.1. Constant state perturbed by small amplitude acoustic waves ${ }^{\text {Remark } 3}$}

The first configuration is a "dimensionalized" version of a Riemann problem taken from [13]. The fluid at stake is endowed with an ideal gas EOS with $\gamma=1.4$. Its density and pressure are initially constant: $\rho^{0}=1 \mathrm{~kg} \cdot \mathrm{m}^{-3}, p^{0}=1 \mathrm{bar}$. Let us also introduce $u^{0}=1 \mathrm{~m} . \mathrm{s}^{-1}$ and a reference Mach number $M^{0}=u^{0} / c^{0}$ with $c^{0} \equiv=\sqrt{p^{0} / \rho^{0}}$. For these values, one obtains $M^{0} \approx 3.2 \times 10^{-3}$. 
As detailed in Table 3 the computational domain of length $L^{0}=1 \mathrm{~m}$ is split in three areas in which the constant velocity $u^{0}$ is perturbed by a term scaling as $O\left(M^{0}\right)$. The initial conditions are thus "well-prepared" according to the definition written in [39, 8].

\begin{tabular}{|c|c|c|c|}
\hline & (zone 1): $x<0.2$ or $x>0.8$ & (zone 2): $x \in[0.2,0.25]$ or $x \in[0.75,0.8]$ & (zone 3): $x \in[0.25,0.75]$ \\
\hline$\rho\left(k g . m^{-3}\right)$ & $\rho^{0}$ & $\rho^{0}$ & $\rho^{0}$ \\
\hline$u\left(m . s^{-1}\right)$ & $u_{L}^{0}=u^{0} \times\left(1-M^{0} / 2\right)$ & $u_{R}^{0}=u^{0} \times\left(1+M^{0} / 2\right)$ & $u_{\mathrm{m}}^{0}=u^{0}$ \\
\hline$p($ bar $)$ & $p^{0}$ & $p^{0}$ & $p^{0}$ \\
\hline
\end{tabular}

Table 3: Dimarco et al Riemann Problem: initial conditions

As time goes on, the four discontinuities of the initial velocity field will produce interacting non-linear waves of small amplitude. For long convective time-scales one expects that these waves fade away and leave a constant velocity field. Here, the ability of the present IMEX approach to seize this almost incompressible state is analyzed.

Once again the proposed IMEX method is compared with the LP-IMEX scheme. The time-steps are given by formulas (4.25) and (5.35) and the computational domain is made of a $10^{3}$ cells mesh. The physical time of the simulation is $T_{\text {end }}=0.05 \times t^{0}$ with $t^{0}=L^{0} / u^{0}$ the convective time-scale of reference. Finally periodic boundary conditions are imposed so that the acoustic waves are constantly re-introduced in the computational domain. Let us end the setting description by mentioning that the shock detector presented in equations 5.36, , 5.37) has been unplugged in this test case.

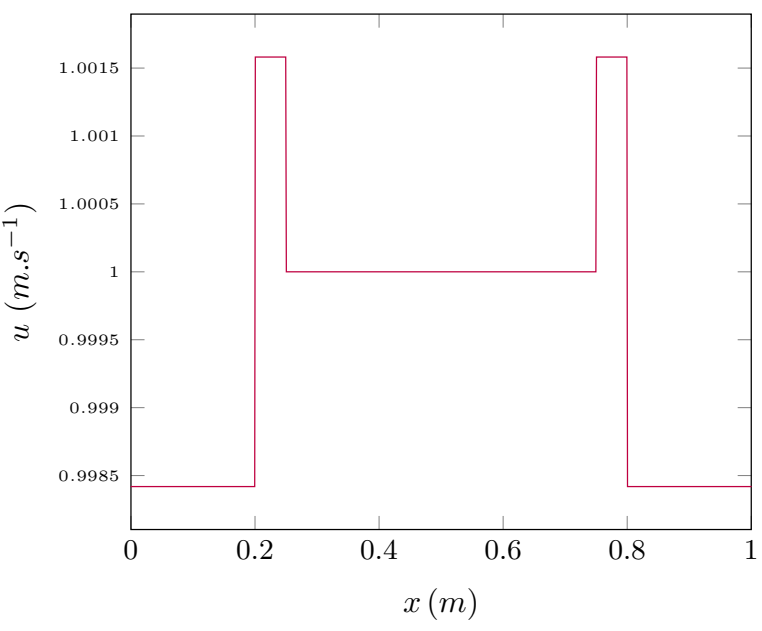

(a) Velocity profile: initial conditions

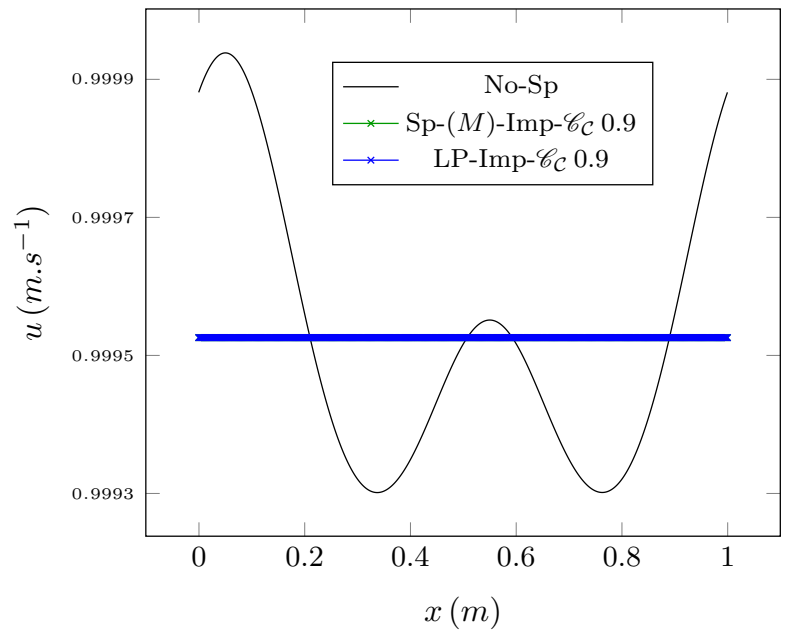

(b) Velocity profile: $T_{\text {end }}=0.05 \mathrm{~s}$

Figure 10: Dimarco's et al Riemann problem: initial conditions and comparison with a full time-explicit scheme

At the final simulation time, Figure 10 a shows the velocity initial distribution while Figure 10b displays the profiles, of the present approach, the LP-IMEX method and a full time-explicit Riemann solver No-Sp. The latter has already been used in subsection 5.2 and is obtained by imposing $\mathscr{E}_{0}^{n}=1$ at every time-step during the simulation. One can notice that both IMEX schemes manage to reach a plateau by the end of the simulation. On the contrary, No-Sp continues to solve all the details produced by the acoustic waves interaction. A closer look at Figure 11a reveals that both time-implicit methods reach the constant value $u^{*} \approx 0.999526 \mathrm{~m} . \mathrm{s}^{-1}$. Such a velocity is consistent with the total momentum conservation since periodic boundary conditions are at stake:

$$
\begin{aligned}
& \int_{\Omega} \rho^{0} u^{*} d \Omega=\int_{\Omega} \rho^{0} u(x, t=0) d \Omega, \\
\Leftrightarrow & \rho^{0} u^{*}=\rho^{0} u^{0}\left[0.4\left(1-M^{0} / 2\right)+0.5+0.1\left(1+M^{0} / 2\right)\right], \\
\Leftrightarrow & u^{*} \approx 0.9995256 .
\end{aligned}
$$




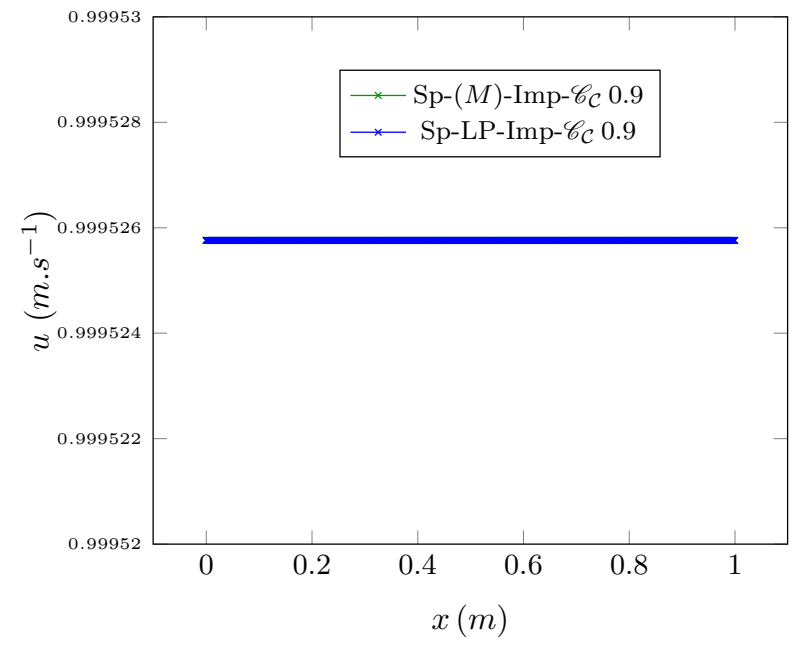

(a) Velocity profile: $T_{\text {end }}=0.05 \mathrm{~s}$, (zoom)

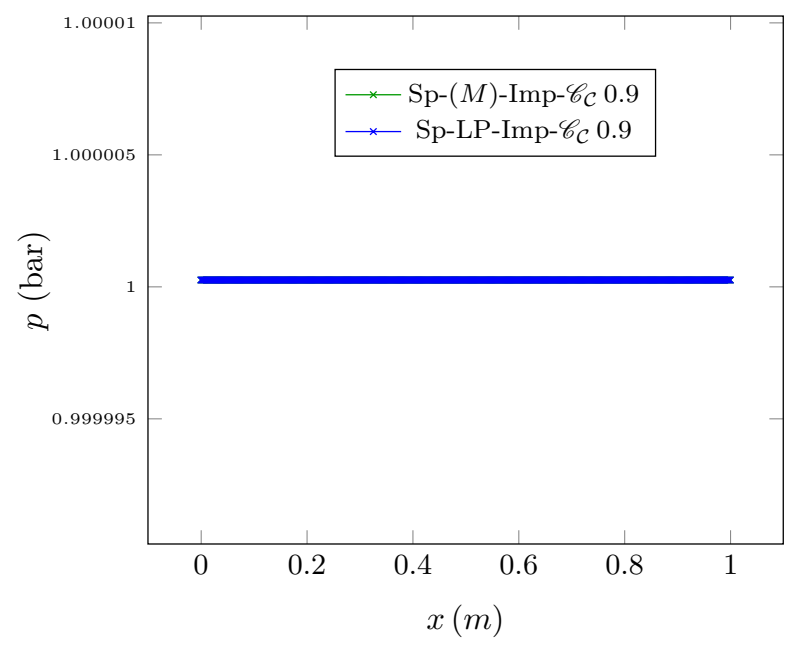

(b) Pressure profile: $T_{\text {end }}=0.05 \mathrm{~s}$, (zoom)

Figure 11: Dimarco's et al Riemann problem: zoom on the constant incompressible state

Finally, a look at Figure $11 \mathrm{~b}$ confirms that the pressure obtained from the IMEX methods also remains constant at the end of the simulation.

\subsection{Weakly Compressible Colliding Pulses}

The last test considered has been taken from [40] and is also treated in [12, 13]. It deals with weakly compressible flows whose solutions are made of long wave acoustic pressure and density pulses. Here, the ability of the present IMEX scheme to follow smooth but fast acoustic waves associated with an intermediate value of the Mach number is assessed. The fluid is endowed with an ideal gas EOS with $\gamma=1.4$. The non-dimensional initial conditions presented in [40] read:

$$
\begin{aligned}
& \bar{\rho}(x, 0)=\bar{\rho}_{0}+\left(\frac{M_{\mathrm{ref}}^{0}}{2}\right) \bar{\rho}_{1}\left(1-\cos \left(\frac{2 \pi x}{L}\right)\right), \bar{\rho}_{0}=0.955, \bar{\rho}_{1}=2, \\
& \bar{u}(x, 0)=-\frac{\bar{u}_{0}}{2} \operatorname{sign}(x)\left(1-\cos \left(\frac{2 \pi x}{L}\right)\right), \bar{u}_{0}=2 \sqrt{\gamma}, \\
& \bar{p}(x, 0)=\bar{p}_{0}+\left(\frac{M_{\mathrm{ref}}^{0}}{2}\right) \bar{p}_{1}\left(1-\cos \left(\frac{2 \pi x}{L}\right)\right), \bar{p}_{0}=1, \bar{p}_{1}=2 \gamma .
\end{aligned}
$$

The Mach number of reference $M_{\text {ref }}^{0}$ has been taken equal to $1 / 11 \approx 9.1 \times 10^{-2}$. The computational domain $\Omega$ is $[-L, L]$ with $L=2 / M_{\text {ref }}^{0}$. Thus, the first-order pulses w.r.t $M_{\text {ref }}^{0}$ evolve with a large space-scale variable $\xi=M_{\text {ref }}^{0} x$. Let us introduce $p_{\text {ref }}^{0}$ (respectively $\rho_{\text {ref }}^{0}, u_{\text {ref }}^{0}$ ) a reference pressure (respectively a reference density, a reference velocity) such that:

$$
\begin{aligned}
& \rho_{\mathrm{ref}}^{0}=1 \mathrm{~kg} \cdot \mathrm{m}^{-3}, u_{\mathrm{ref}}^{0}=1 \mathrm{~m} \cdot \mathrm{s}^{-1}, \\
& p_{\mathrm{ref}}^{0}=\frac{\rho_{\mathrm{ref}}^{0} \bar{\rho}_{0}}{\gamma}\left(\frac{u_{\mathrm{ref}}^{0} \bar{u}_{0}}{M_{\mathrm{ref}}^{0}}\right)^{2} .
\end{aligned}
$$

The reference pressure $p_{\text {ref }}^{0}$ has been set such that the exact maximal value of the initial Mach number is of the order of $M_{\mathrm{ref}}^{0}$. The dimensional initial conditions then write: $\rho(x, 0)=\rho_{\mathrm{ref}}^{0} \bar{\rho}(x, 0), u(x, 0)=u_{\mathrm{ref}}^{0} \bar{u}(x, 0)$ and $p(x, 0)=p_{\mathrm{ref}}^{0} \bar{p}(x, 0)$. Figure 12 and Figure 13 display the pressure and velocity profiles at two physical times. The first one captures the time when the two pulses collide producing an over-pressure peak at the center of the computational domain. The second one corresponds to the instant where the two pressure pulses have separated again. 


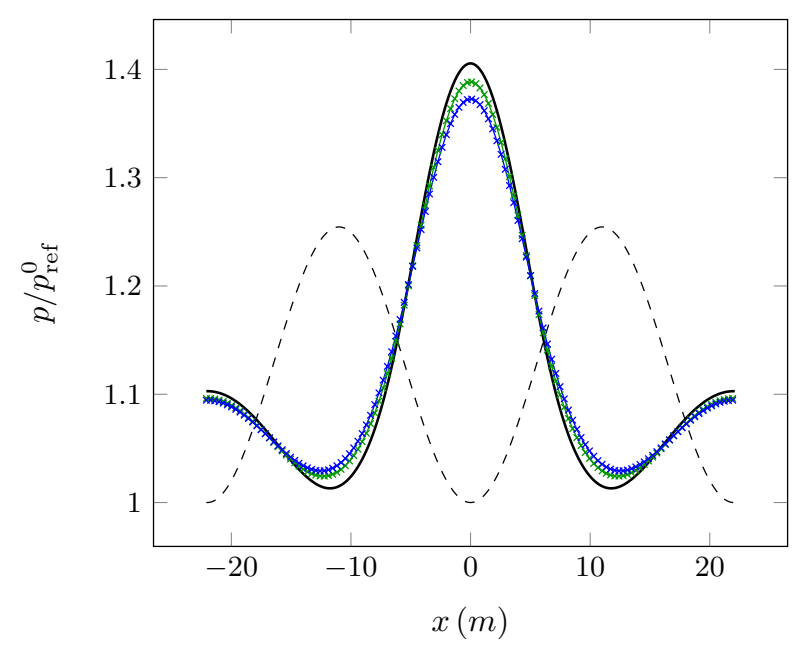

(a) Pressure profile: $t=3.78 \times 10^{-1} \mathrm{~s}$

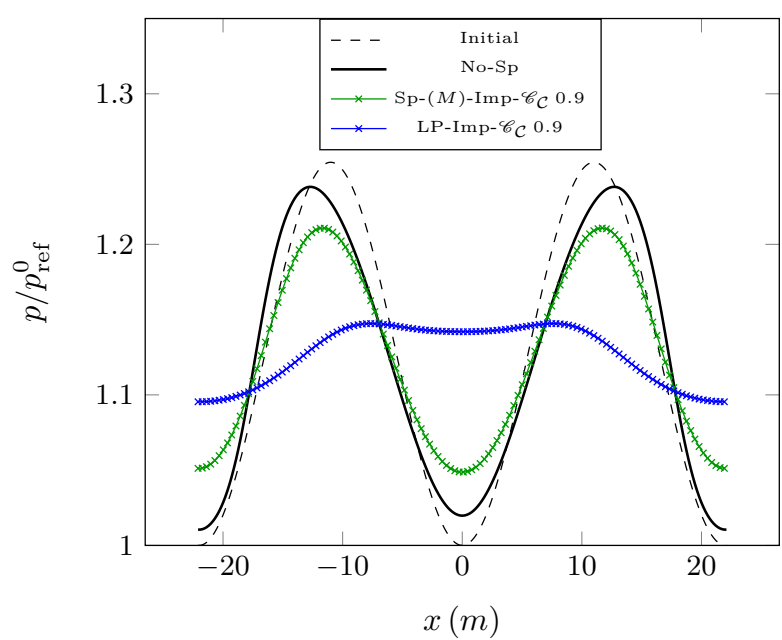

(b) Pressure profile: $t=8.41 \times 10^{-1} \mathrm{~s}$

Figure 12: Pressure: just after the pulses super-position (a), once the pulses have separated again (b)

The present IMEX approach with no shock detector, is compared with the LP-IMEX and No-Sp schemes. The non-dimensional initial conditions have been added as a dashed line in order to observe the diffusion and dispersion introduced w.r.t the initial pulses.

In Figure 12a, one can notice that both IMEX methods manage to catch the over-pressure peak although they are slightly more diffusive than the full time-explicit scheme. However, in Figure $12 \mathrm{~b}$, the full time-implicit discretization of the pressure gradient in the LP-Imp approach combined with a CFL condition based on $u$ completely diffuses the re-appearing pressure pulses. On the contrary, in the case of the present IMEX method, one can notice that $\mathscr{E}_{0}$ remains of order $M_{\text {ref }}^{0}$ during most of the simulation. For example, at time $t=8.41 \times 10^{-1} s, M_{\max }^{n}=7.4 \times 10^{-2}=\mathscr{E}_{0}^{n}$. The intermediate value of $\mathscr{E}_{0}$ is enough to produce considerably lower time-steps which are more appropriate to follow the long acoustic waves. As a result, even if it is more diffused compared with the full-time explicit scheme, the global shape of the pressure pulses is retrieved. However, the present approach suffers from the same drawback as the one noticed in [40]: in the vicinity of the locations $x \approx \pm 18.5 \mathrm{~m}$, the pressure gradient stiffens such that the long-length scale acoustic wave hypothesis no longer holds, and the time-implicit discretization of the acoustic subsystem considerably smears the appearing discontinuities. In any case, a specific additional work should be undertaken if one is interested in capturing the fast acoustic dynamics related to first-order pressure terms w.r.t $M_{\text {ref }}^{0}$. 


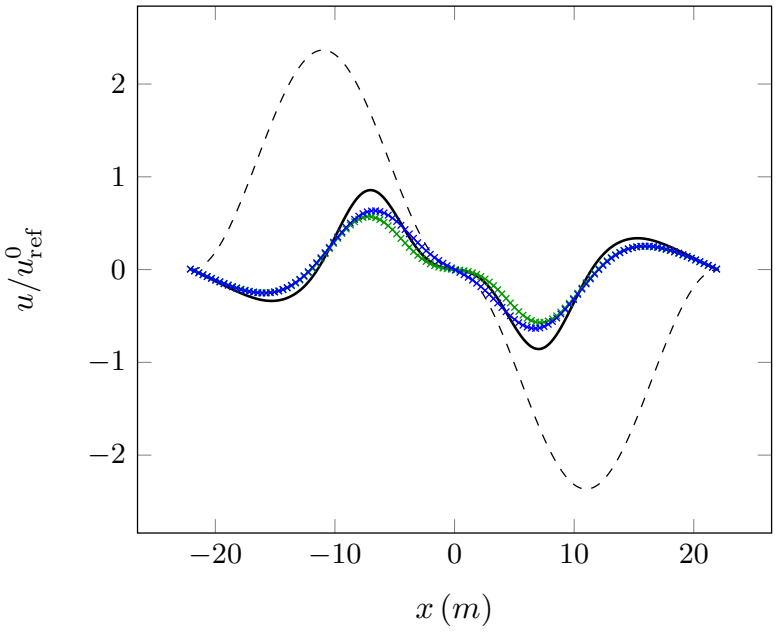

(a) Velocity profile: $t=3.78 \times 10^{-1} \mathrm{~s}$

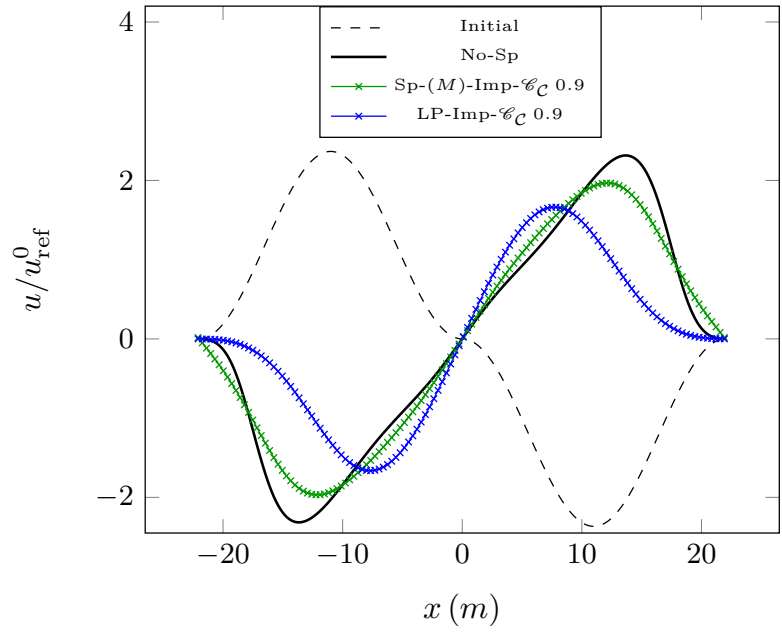

(b) Velocity profile: $t=8.41 \times 10^{-1} \mathrm{~s}$

Figure 13: Velocity: just after the pulses super-position (a), once the pulses have separated again (b)

\section{Conclusion}

The present work focuses on the derivation of an IMEX version of the Mach-sensitive fractional step introduced in [19, 20]. The time-implicit scheme derived for the acoustic subsystem is based on the non-conservative dynamics of strong Riemann invariants provided by the relaxation schemes framework. Although such a technique stems from the Lagrange-Projection theory [18, 29], it can be extended to the present splitting because of density time invariance in the acoustic sub-step and the piece-wise constant structure of the computed solution. The resulting IMEX scheme is simple, the implicit part being inverted by hand, and conservative by construction. What is more, the maximum principle preservation for purely convected quantities holds under a non-restrictive condition for the time-explicit convective flux.

A low Mach number shock tube involving an ideal gas thermodynamics and small pressure jumps has highlighted a trade-off in the use of IMEX schemes. Indeed, if one is interested by pressure jumps through shock waves, then using an IMEX scheme with $\mathscr{C}_{E} \gg 1$ might be inappropriate in terms of accuracy. Besides, the gain in CPU time is not sufficient to compensate the implicit diffusion and dispersion errors: for the pressure variable, IMEX schemes are still less efficient than full time-explicit ones. However, any quantities varying only through material waves should be depicted more efficiently and with a sufficient accuracy.

When the thermodynamics becomes stiffer, high amplitude pressure jumps can occur in low-Mach number flows. This is for example the case during water-hammer events. Then, one can complete the splitting parameter $\mathscr{E}_{0}$ with a basic "shock detector" which enforces the initial IMEX scheme associated with a convective-like CFL condition to turn into a fully time-explicit approximate Riemann solver related to an acoustic-like CFL condition. Hence, the splitting parameter allows to select the acoustic-flux time-discretization and in the same time the time-step adapted to the physical-scale looked at. The application of such a strategy to a double Riemann problem endowed with a stiffened gas EOS has shown that, contrary to the IMEX version of the Lagrange-Projection fractional step, the present IMEX approach is able to accurately follow stiff rarefaction and shock waves even in low velocity regions.

Besides, when the fluid is at rest, the proposed IMEX scheme is able to filter out small amplitude acoustic waves of no interest in order to retrieve the underlying constant state. The present IMEX approach has also been tried on intermediate configurations in which smooth and large-scale acoustic waves associated with first order terms w.r.t the flow Mach number propagate and need to be followed. The proposed method provides lower time-steps than the one produced by the LP-IMEX scheme. It is thus able to capture the global shape of the pressure colliding or re-appearing pulses but considerably smears the solution in regions where the pressure gradient stiffens.

Finally, the different test cases presented in this study show that the present IMEX scheme is stable, whatever the Mach number, under $\mathscr{C}_{C} \approx 1$; with $\mathscr{C}_{C}$ the Courant number based on the eigenvalues of the convective subsystem $C$. 
Even if it is not yet rigorously proved, the Mach-uniform- $\mathscr{C}_{C}$ stability property seems to hold.

The fact is that, at a given instant, the computational domain can feature subsonic areas as well as sonic or supersonic ones. Thus, the spatial dependence of the splitting parameter could also been examined. Indeed, provided that the consistency of the overall fractional step is not deteriorated, such a spatial dependence would allow to capture local fluctuations of the Mach number, and the present approach to react more finely. In addition, some improvements regarding the construction of the "shock detector" could also be proposed.

\section{Acknowledgements}

The first author received a financial support through the EDF-CIFRE contract 0561-2015. Computational facilities were provided by EDF.

The authors would also like to thank the reviewers who have spent time reading this paper. Indeed, the remarks and questions they have pointed out have considerably allowed the present work to be improved. 


\section{Appendix A. Discrete Preservation of the Contact Discontinuity Riemann Invariants}

Before focusing on the preservation of the contact discontinuity Riemann invariants property, let us have a look on the discrete momentum equation related to the flux 4.22.

$$
\begin{aligned}
& \text { Assume that: } u_{i}^{n+}=\frac{R_{i}^{n+}+W_{i}^{n+}}{2} \text {,then: } \\
& u_{i}^{n+1}=\frac{R_{i}^{n+1}+W_{i}^{n+1}}{2} \\
& \Leftrightarrow \frac{u_{i}^{n+1}-u_{i}^{n+}}{\Delta t}=\frac{1}{2}\left[\frac{R_{i}^{n+1}-R_{i}^{n+}}{\Delta t}+\frac{W_{i}^{n+1}-W_{i}^{n+}}{\Delta t}\right] \\
& \Leftrightarrow \frac{u_{i}^{n+1}-u_{i}^{n+}}{\Delta t}=-\frac{\left(1-\left(\mathscr{E}_{0}^{n}\right)^{2}\right)\left(a_{\mathcal{A}}\right)^{n+} \tau_{i}^{n+}}{2}\left[\frac{R_{i}^{n+1}-R_{i-1}^{n+1}}{\Delta x}-\frac{W_{i+1}^{n+1}-W_{i}^{n+1}}{\Delta x}\right] \\
& \overbrace{\Leftrightarrow}^{\rho_{i}^{n+1}=\rho_{i}^{n+}} \frac{\rho_{i}^{n+1} u_{i}^{n+1}-\rho_{i}^{n+} u_{i}^{n+}}{\Delta t}+\frac{\left(1-\left(\mathscr{E}_{0}\right)^{2}\right)\left(a_{\mathcal{A}}\right)^{n+}}{\Delta x}\left[\frac{R_{i}^{n+1}-W_{i+1}^{n+1}}{2}-\frac{R_{i-1}^{n+1}-W_{i}^{n+1}}{2}\right]=0 \\
& \Leftrightarrow \frac{\rho_{i}^{n+1} u_{i}^{n+1}-\rho_{i}^{n+} u_{i}^{n+}}{\Delta t}+\left(1-\left(\mathscr{E}_{0}^{n}\right)^{2}\right) \frac{\left(p_{\mathcal{A}}^{*}\right)_{i+1 / 2}^{n+1}-\left(p_{\mathcal{A}}^{*}\right)_{i-1 / 2}^{n+1}}{\Delta x}=0, \\
& \text { with: }\left(p_{\mathcal{A}}^{*}\right)_{i+1 / 2}^{n+1}=\left(a_{\mathcal{A}}\right)^{n+} \frac{R_{i}^{n+1}-W_{i+1}^{n+1}}{2} .
\end{aligned}
$$

Thus:

$$
\begin{aligned}
& u_{i}^{n+}=\frac{R_{i}^{n+}+W_{i}^{n+}}{2}, \text { and } u_{i}^{n+1}=\frac{R_{i}^{n+1}+W_{i}^{n+1}}{2} \Leftrightarrow \\
& \left.u_{i}^{n+}=\frac{R_{i}^{n+}+W_{i}^{n+}}{2}, \text { and } \frac{(\rho u)_{i}^{n+1}-(\rho u)_{i}^{n+}}{\Delta t}+\left(1-\left(\mathscr{E}_{0}^{n}\right)^{2}\right)\right) \frac{\left(p_{\mathcal{A}}^{*}\right)_{i+1 / 2}^{n+1}-\left(p_{\mathcal{A}}^{*}\right)_{i-1 / 2}^{n+1}}{\Delta x}=0 .
\end{aligned}
$$

Then, if $u_{i}^{n+}=\left(R_{i}^{n+}+W_{i}^{n+}\right) / 2$, solving the momentum equation is strictly equivalent to directly setting $u_{i}^{n+1}=$ $\left(R_{i}^{n+1}+W_{i}^{n+1}\right) / 2$.

\section{Appendix A.1. Discrete Preservation of the Riemann Invariants of a Contact Discontinuity}

Consider an equation of state such that:

$$
(\rho \varepsilon)^{\mathrm{EOS}}(\rho, p)=C(p) \rho+B(p),
$$

with $p \rightarrow C(p)$ and $p \rightarrow B(p)$ smooth functions such as $(\rho \varepsilon)_{\mid \rho}^{\mathrm{EOS}}: p \rightarrow C(p) \rho+B(p)$ is injective on the domain of definition of $p$. Formula (A.3] belongs to the "T1-class" of EOS introduced in [31]. One can notice that the stiffened gas EOS is included in this category. In the sequel, the exact invariance of velocity and pressure in the case of an isolated contact wave described in subsection 5.1.2 is checked.

\section{Appendix A.1.1. Acoustic Sub-step}

Assume that at the end of the convective sub-step, the isolated contact discontinuity has been preserved:

$$
\begin{aligned}
& \forall i: u_{i}^{n+}=u^{0}, p_{i}^{n+}=p^{0}, \\
\Rightarrow & \forall i: W_{i}^{n+}=u^{0}-\frac{p^{0}}{a_{\mathcal{A}}^{n+}}=W^{0, n+}, R_{i}^{n+}=u^{0}+\frac{p^{0}}{a_{\mathcal{A}}^{n+}}=R^{0, n+} .
\end{aligned}
$$

Considering the discrete dynamics of $W$ and $R$ written in 4.20 coupled with the transparent boundary conditions $W_{N_{\text {cells }}+1}^{n+}=W_{N_{\text {cells }}}^{n+}$ and $W_{0}^{n+}=W_{1}^{n+}$, one can easily see that:

$$
\forall i: W_{i}^{n+1}=W^{0, n+}, R_{i}^{n+1}=R^{0, n+} .
$$


Please note that the constant relaxation hypothesis $\left(a_{\mathcal{A}}\right)^{n}=K \max _{i \in\left[1, N_{\text {cells }}\right]}\left(\rho_{i}^{n}\left(c_{\mathcal{A}}\right)_{i}^{n}\right), K>1$, plays an important role here since a local value of $a_{\mathcal{A}}$ would have been sensible to the density discontinuity preventing $W$ and $R$ to remain constant. Thus, property (A.5) cannot be guaranteed in this case. Then, $\left(p_{\mathcal{A}}^{*}\right)_{i+1 / 2}^{n+1}=\left(p_{\mathcal{A}}^{*}\right)_{i-1 / 2}^{n+1}=a_{\mathcal{A}}^{n+}\left(R^{0, n+}-W^{0, n+}\right) / 2$ and $\left(u_{\mathcal{A}}^{*}\right)_{i+1 / 2}^{n+1}=\left(u_{\mathcal{A}}^{*}\right)_{i-1 / 2}^{n+1}=\left(R^{0, n+}+W^{0, n+}\right) / 2$ such that the discrete fluctuation of the time-implicit acoustic flux is null. The mass, momentum and the energy equations thus become:

$$
\begin{array}{cl}
\frac{\rho_{i}^{n+1}-\rho_{i}^{n+}}{\Delta t}=0, & \\
& \rho_{i}^{n+1}=\rho_{i}^{n+}, \\
\rho_{i}^{n+} \frac{u_{i}^{n+1}-u_{i}^{n+}}{\Delta t}=0, & u_{i}^{n+1}=u_{i}^{n+}=u^{0}, \\
\rho_{i}^{n+} \frac{\left(\varepsilon_{i}^{n+1}-\varepsilon_{i}^{n+}\right)+\left(\left(u_{i}^{n+1}\right)^{2} / 2-\left(u_{i}^{n+}\right)^{2} / 2\right)}{\Delta t}=0 . & (\rho \varepsilon)^{\operatorname{EOS}}\left(\rho_{i}^{n+}, p_{i}^{n+1}\right)=(\rho \varepsilon)^{\operatorname{EOS}}\left(\rho_{i}^{n+}, p_{i}^{n+}\right) .
\end{array}
$$

Since $(\rho \varepsilon)_{\mid \rho}^{\mathrm{EOS}}: p \rightarrow C(p) \rho+B(p)$ is injective, $p_{i}^{n+1}=p_{i}^{n+}=p^{0}$ and the acoustic sub-step exactly preserves the velocity and pressure uniform profiles.

\section{Appendix A.1.2. Convective Sub-step}

The convective flux associated to subsystem $C$ is obtained using the same kind of relaxation method. Details are given in [19, 20]. The convective flux formula at face $i+1 / 2$ reads:

$$
\mathbf{H}_{C i+1 / 2}^{n}= \begin{cases}\left(\mathbf{F}_{C}\right)_{i}^{n} & \text { if } u_{i}^{n}-\mathscr{E}_{0}^{n}\left(a_{C}^{n}\right)_{i+1 / 2} \tau_{i}^{n}>0 \\ \left(\mathbf{F}_{C}\right)_{i+1 / 2}^{*, n} & \text { if } u_{i}^{n}-\mathscr{E}_{0}^{n}\left(a_{C}^{n}\right)_{i+1 / 2} \tau_{i}^{n} \leq 0<\left(u_{C}^{*}\right)_{i+1 / 2}^{n} \\ \left(\mathbf{F}_{C}\right)_{i+1 / 2}^{* * n} & \text { if }\left(u_{C}^{*}\right)_{i+1 / 2}^{n} \leq 0<u_{i+1}^{n}+\mathscr{E}_{0}^{n}\left(a_{C}^{n}\right)_{i+1 / 2} \tau_{i+1}^{n} \\ \left(\mathbf{F}_{C}\right)_{i+1}^{n} & \text { if } u_{i+1}^{n}+\mathscr{E}_{0}^{n}\left(a_{C}^{n}\right)_{i+1 / 2} \tau_{i+1}^{n} \leq 0 \\ \left(a_{C}^{n}\right)_{i+1 / 2}= & K \max \left(\rho_{i}^{n}\left(c_{C}\right)_{i}^{n}, \rho_{i+1}^{n}\left(c_{C}\right)_{i+1}^{n}\right), K>1 \\ \mathbf{F}_{C}(\mathbf{U})= & {\left[\rho u, \rho u^{2}+\mathscr{E}_{0}^{2} p,\left(\rho e+\mathscr{E}_{0}^{2} p\right) u\right]^{T},}\end{cases}
$$

where $\left(a_{C}^{n}\right)_{i+1 / 2}=K \max \left(\rho_{i}^{n}\left(c_{C}\right)_{i}^{n}, \rho_{i+1}^{n}\left(c_{C}\right)_{i+1}^{n}\right), K>1$, the discrete convective relaxation constant fulfilling the convective subcharacteristic condition, i.e. $a_{C}>\rho c_{C}$, throughout the whole computational domain and with:

$$
\begin{aligned}
& \left(\mathbf{F}_{C}\right)_{i+1 / 2}^{*, n}=\left[\begin{array}{c}
\left(\rho_{C}^{*}\right)_{i}^{n}\left(u_{C}^{*}\right)_{i+1 / 2}^{n} \\
\left(\rho_{C}^{*}\right)_{i}^{n}\left(\left(u_{C}^{*}\right)_{i+1 / 2}^{n}\right)^{2}+\left(\mathscr{E}_{0}^{n}\right)^{2}\left(\Pi_{C}^{*}\right)_{i+1 / 2}^{n} \\
\left(\left(\rho_{C}^{*} e_{C}^{*}\right)_{i}^{n}+\left(\mathscr{E}_{0}^{n}\right)^{2}\left(\Pi_{C}^{*}\right)_{i+1 / 2}^{n}\right)\left(u_{C}^{*}\right)_{i+1 / 2}^{n}
\end{array}\right], \\
& \left(\mathbf{F}_{C}\right)_{i+1 / 2}^{* * n}=\left[\begin{array}{c}
\left(\rho_{C}^{*}\right)_{i+1}^{n}\left(u_{C}^{*}\right)_{i+1 / 2}^{n} \\
\left(\rho_{C}^{*}\right)_{i+1}^{n}\left(\left(u_{C}^{*}\right)_{i+1 / 2}^{n}\right)^{2}+\left(\mathscr{E}_{0}^{n}\right)^{2}\left(\Pi_{C}^{*}\right)_{i+1 / 2}^{n} \\
\left(\left(\rho_{C}^{*} e_{C}^{*}\right)_{i+1}^{n}+\left(\mathscr{E}_{0}^{n}\right)^{2}\left(\Pi_{C}^{*}\right)_{i+1 / 2}^{n}\right)\left(u_{C}^{*}\right)_{i+1 / 2}^{n}
\end{array}\right], \\
& \left(u_{C}^{*}\right)_{i+1 / 2}^{n}=\frac{u_{i+1}^{n}+u_{i}^{n}}{2}-\frac{\mathscr{E}_{0}^{n}}{2\left(a_{C}\right)_{i+1 / 2}^{n}}\left(p_{i+1}^{n}-p_{i}^{n}\right), \\
& \left(\mathscr{E}_{0}^{n}\right)^{2}\left(\Pi_{C}^{*}\right)_{i+1 / 2}^{n}=\left(\mathscr{E}_{0}^{n}\right)^{2} \frac{p_{i+1}^{n}+p_{i}^{n}}{2}-\frac{\mathscr{E}_{0}^{n}\left(a_{C}\right)_{i+1 / 2}^{n}}{2}\left(u_{i+1}^{n}-u_{i}^{n}\right), \\
& \left(\rho_{C}^{*}\right)_{k}^{n}=1 /\left(\tau_{k, C}^{*}\right)^{n},\left(\tau_{k, C}^{*}\right)^{n}=\tau_{k}^{n}+\frac{(-1)^{J_{k}+1}}{\mathscr{E}_{0}^{n}\left(a_{C}\right)_{i+1 / 2}^{n}}\left(\left(u_{C}^{*}\right)_{i+1 / 2}^{n}-u_{k}^{n}\right), \\
& \left(e_{C}^{*}\right)_{k}^{n}=e_{k}^{n}+\mathscr{E}_{0}^{n} \frac{(-1)^{J_{k}}}{\left(a_{C}\right)_{i+1 / 2}^{n}}\left(\left(\Pi_{C}^{*} u_{C}^{*}\right)_{i+1 / 2}^{n}-p_{k}^{n} u_{k}^{n}\right), \\
& k \in\{i, i+1\}, J_{i}=1, J_{i+1}=2 .
\end{aligned}
$$


The time-explicit scheme solving the convective subsystem then reads:

$$
\mathbf{U}_{i}^{n+}=\mathbf{U}_{i}^{n}-\frac{\Delta t}{\Delta x}\left(\mathbf{H}_{C_{i+1 / 2}^{n}}^{n}-\mathbf{H}_{C_{i-1 / 2}^{n}}^{n}\right)
$$

In the case of an isolated contact discontinuity with $\underline{u^{0}>0}$, the convective flux writes:

$$
\mathbf{H}_{C_{i+1 / 2}^{n, \text { Contact }}}=\left[\begin{array}{c}
\rho_{i}^{n} u^{0} \\
\rho_{i}^{n}\left(u^{0}\right)^{2}+\left(\mathscr{E}_{0}\right)^{2} p^{0} \\
(\rho \varepsilon)^{\mathrm{EOS}}\left(\rho_{i}^{n}, p^{0}\right) u^{0}+\rho_{i}^{n} \frac{\left(u^{0}\right)^{3}}{2}+\left(\mathscr{E}_{0}^{n}\right)^{2} p^{0} u^{0}
\end{array}\right] .
$$

The mass, momentum and energy dynamics then read:

$$
\begin{aligned}
& \frac{\rho_{i}^{n+}-\rho_{i}^{n}}{\Delta t}+u^{0} \frac{\rho_{i}^{n}-\rho_{i-1}^{n}}{\Delta x}=0, \\
& \frac{\rho_{i}^{n+} u_{i}^{n+}-\rho_{i}^{n} u^{0}}{\Delta t}+\left(u^{0}\right)^{2} \frac{\rho_{i}^{n}-\rho_{i-1}^{n}}{\Delta x}=0, \\
& \frac{(\rho \varepsilon)^{\mathrm{EOS}}\left(\rho_{i}^{n+}, p_{i}^{n+}\right)-(\rho \varepsilon)^{\mathrm{EOS}}\left(\rho_{i}^{n}, p^{0}\right)}{\Delta t}+\frac{1}{2} \frac{\rho_{i}^{n+}\left(u_{i}^{n+}\right)^{2}-\rho_{i}^{n}\left(u^{0}\right)^{2}}{\Delta t} \\
& +u^{0} \frac{(\rho \varepsilon)^{\mathrm{EOS}}\left(\rho_{i}^{n}, p^{0}\right)-(\rho \varepsilon)^{\mathrm{EOS}}\left(\rho_{i-1}^{n}, p^{0}\right)}{\Delta x}+\frac{\left(u^{0}\right)^{3}}{2} \frac{\rho_{i}^{n}-\rho_{i-1}^{n}}{\Delta x}=0 .
\end{aligned}
$$

By rewriting $\rho_{i}^{n+} u_{i}^{n+}-\rho_{i}^{n} u^{0}$ as $\rho_{i}^{n+}\left(u_{i}^{n+}-u^{0}\right)+\left(\rho_{i}^{n+}-\rho_{i}^{n}\right) u^{0}$ and using the discrete mass equation, the momentum equation can be simplified:

$$
\rho_{i}^{n+} \frac{u_{i}^{n+}-u^{0}}{\Delta t}=0 \Rightarrow u_{i}^{n+}=u^{0}
$$

The kinetic part in the discrete energy equation then vanishes by factorizing by $\left(u^{0}\right)^{2} / 2$ and using, once again, the discrete mass equation. Injecting formula A.3, one obtains:

$$
\frac{C\left(p_{i}^{n+}\right) \rho_{i}^{n+}+B\left(p_{i}^{n+}\right)-\left(C\left(p^{0}\right) \rho_{i}^{n}+B\left(p^{0}\right)\right)}{\Delta t}+u^{0} \frac{C\left(p^{0}\right)\left(\rho_{i}^{n}-\rho_{i-1}^{n}\right)}{\Delta x}=0 .
$$

The linear behavior of $(\rho \varepsilon)_{\mid p}^{\mathrm{EOS}}: \rho \rightarrow C(p) \rho+B(p)$ as well as the fact that $C(p)=C\left(p^{0}\right)$ is a constant in this configuration, play an important role. Indeed, it allows to retrieve the discrete mass equation by factorizing by $C\left(p^{0}\right)$. Finally, one obtains:

$$
\begin{aligned}
& \frac{C\left(p_{i}^{n+}\right) \rho_{i}^{n+}+B\left(p_{i}^{n+}\right)-\left(C\left(p^{0}\right) \rho_{i}^{n+}+B\left(p^{0}\right)\right)}{\Delta t}=0 \\
\Leftrightarrow & \frac{(\rho \varepsilon)^{\operatorname{EOS}}\left(\rho_{i}^{n+}, p_{i}^{n+}\right)-(\rho \varepsilon)^{\mathrm{EOS}}\left(\rho_{i}^{n+}, p^{0}\right)}{\Delta t}=0 .
\end{aligned}
$$

Using the fact that $(\rho \varepsilon)_{\mid \rho_{i}^{n+}}^{\mathrm{EOS}}: p \rightarrow C(p) \rho_{i}^{n+}+B(p)$ is injective, it results in $p_{i}^{n+}=p^{0}$.

\section{Appendix B. Stability Analysis}

This appendix deals with the definition of a stability criterion for the proposed IMEX scheme. It focuses on the isolated contact discontinuity test case discussed in subsection 5.1.2 As shown in Figure D.15 and Figure D.16 in Appendix D, the present splitting seems to suffer from instabilities when the convective Courant number $\mathscr{C}_{|u|}$ goes over a certain threshold. In the following, the dependence to the Mach number $M$ of such a threshold is examined. One can notably wonder whether the time-explicit CFL condition $\mathscr{C}_{|u|}=M /(1+M) \mathscr{C}_{E}$ is retrieved as $M$ tends toward zero.

In the sequel, Appendix B.1 describes a pragmatic way to measure stable Courant numbers while Appendix B.2 endeavors to derive a von Neumann stability analysis predicting stable Courant numbers analytically. 


\section{Appendix B.1. Empirical Stable Courant Numbers}

As stated in Proposition 4.3 for a sufficiently simple shape of the equation of state, velocity and pressure are supposed to be left constant by the overall scheme from one time-step to another. Nonetheless, truncation errors on double-precision floating-point numbers can be amplified by the scheme's instability and lead to the crash of the simulation. Thus, the selected criterion to detect the instability appearance is: $\max _{i}\left(\left|p_{i}^{n}-p^{0}\right|\right) / p^{0}>\eta$ with $\eta=10^{-7}$ which is nearly the single-precision for floating-point numbers. Besides at the beginning of each calculation a ramp of CFL is enforced so that the targeted Courant number is reached after 200 time-steps, which corresponds to a propagation of $0.1 \mathrm{~m}$ of the exact contact discontinuity. If the calculation ends without triggering the above pressure stability criterion then the same calculation is launched again on a five times finer mesh of $5 \times 10^{3}$ cells in order to make sure that the numerical diffusion has not damped the instability appearance.

In Figure B.14 the curve labeled Sp- $(M)$ gathers the different points resulting from the above stable convective Courant number research. Recall that in this test case the velocity is given by $u^{0}=M_{\min } c_{R}^{0}$, with $c^{0, R}=\sqrt{\left(\gamma p^{0}\right) / \rho_{R}^{0}}$ the maximal sound speed and $M_{\min }$ the minimal Mach number of the flow used as an input parameter here. Hence the stable Courant number upper bound is displayed as a function of $M_{\min }$. The latter starts from $M_{\min }=1, u^{0}=335\left(m \cdot s^{-1}\right)$ and decreases until $M_{\min }=10^{-4}, u^{0}=0.0335\left(m . s^{-1}\right)$.

(a)

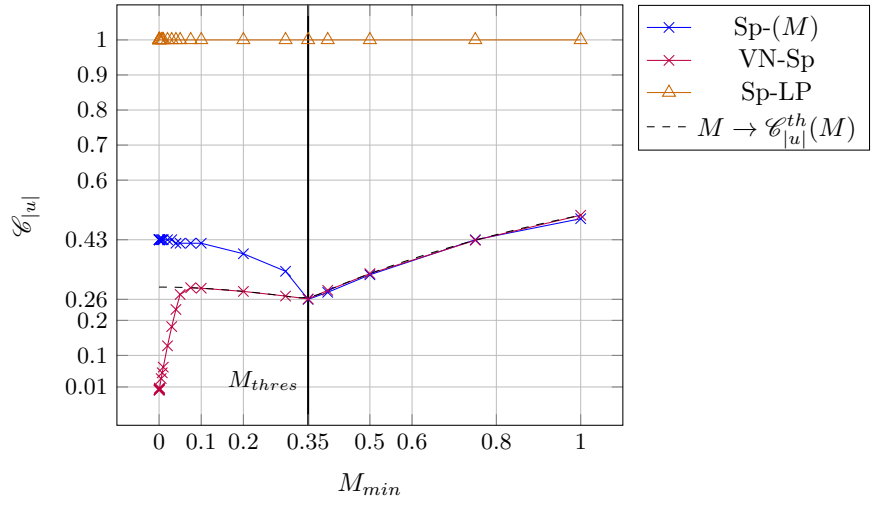

(b)

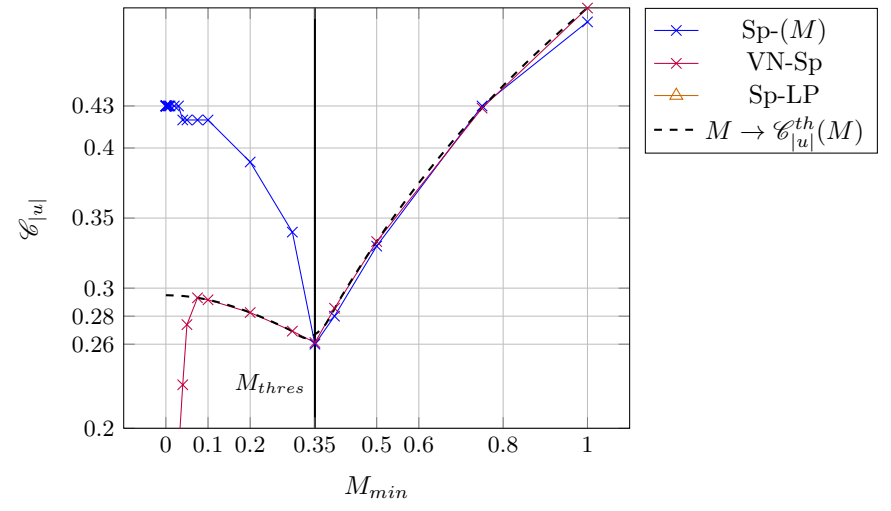

Figure B.14: Evaluation of an upper bound for the stable convective Courant number $\mathscr{C}_{|u|}$ as function of the Mach number $M_{m i n}$ : global view (a) and zoom in the region $\mathscr{C}_{|u|} \in[0.2,0.5]$ (b)

One can observe that for $M_{\min } \approx 1, \mathscr{E}_{0} \approx 1$ so that the full Euler system is brought back into the time-explicit convective subsystem. The latter is thus bound by the classical CFL condition $\mathscr{C}_{E} \approx 1$. As $\mathscr{C}_{|u|}=M /(1+M) \mathscr{C}_{E}$, this leads to $\mathscr{C}_{|u|} \approx 0.5$ for $M_{\text {min }} \approx 1$ which is observed here. Then, as the Mach number decreases, the contribution of the time-implicit part of the acoustic subsystem becomes more and more active. It results in a drop of the Euler timeexplicit CFL condition until $M_{\min } \approx 0.35$ where $\mathscr{C}_{|u|}=0.26$.

An attempt at explaining this decline is given in the following. First, as it will be shown, this behavior might be related to the gap between the definition of $\mathscr{C}_{|u|}$ and the Courant number involving the largest eigenvalues of the convective subsystem as given in 4.4 .

$$
\begin{aligned}
& \mathscr{C}_{C}=\frac{\Delta t}{\Delta x}\left(\left|u^{0}\right|+\mathscr{E}_{0}^{n} c_{C}^{0, R}\right) \\
& \text { with: } c_{C}^{0, R}=c_{C}\left(\rho_{R}^{0}, p^{0}\right) .
\end{aligned}
$$


One can notice that:

$$
\begin{aligned}
& \mathscr{C}_{|u|}=\left(1+\mathscr{E}_{0} \frac{c_{C}^{0, R}}{\left|u^{0}\right|}\right)^{-1} \mathscr{C}_{C}=\left(1+\frac{\mathscr{E}_{0}^{n}}{M_{\min }} \frac{c_{C}^{0, R}}{c^{0, R}}\right)^{-1} \mathscr{C}_{C}, \\
& \text { since: }\left|u^{0}\right|=M_{\min } c^{0, R}, \\
& \text { and: } \frac{c_{C}^{0, R}}{c^{0, R}}=\sqrt{\left(\mathscr{E}_{0}\right)^{2}} \frac{\gamma-1}{\gamma}+\frac{1}{\gamma} \in[1 / \gamma, 1] .
\end{aligned}
$$

Besides, according to formula 4.19):

$$
\begin{aligned}
\mathscr{E}_{0} n=\min \left(M_{\text {max }}^{n}, 1\right) & =\min \left(\left|u^{0}\right| / c^{0, L}, 1\right) \\
& =\min \left(M_{\min } \sqrt{\frac{\rho_{L}^{0}}{\rho_{R}^{0}}}, 1\right), \\
\text { then for } M_{\text {min }} \geq M_{\text {thres }} & =\sqrt{\frac{\rho_{R}^{0}}{\rho_{L}^{0}}}: \mathscr{E}_{0}^{n}=1, \\
\text { and for } M_{\text {min }}<M_{\text {thres }} & =\sqrt{\frac{\rho_{R}^{0}}{\rho_{L}^{0}}}: \mathscr{E}_{0}^{n}=M_{\text {min }} / M_{\text {thres }} .
\end{aligned}
$$

It results that:

$$
\mathscr{C}_{|u|}= \begin{cases}\left(1+\frac{1}{M_{\text {min }}}\right)^{-1} \mathscr{C}_{C}, & \text { if } M_{\text {min }} \geq M_{\text {thres }}, \\ \left(1+\frac{1}{M_{\text {thres }}} \sqrt{\left.\left(\frac{M_{\text {min }}}{M_{\text {thres }}}\right)^{2} \frac{\gamma-1}{\gamma}+\frac{1}{\gamma}\right)^{-1}} \mathscr{C}_{C},\right. & \text { otherwise. }\end{cases}
$$

Define $M_{\min } \rightarrow \mathscr{C}_{|u|}^{\text {th }}\left(M_{\min }\right)$, the continuous function described by formula B.4. This function has been plotted as a dashed black line in Figure B.14

For every $M_{\min }$ between $M_{\text {thres }}$ and 1 the comparison between $\mathscr{C}_{|u|}^{\text {th }}$ and the measured stable convective Courant number is fair: the cut-off $M_{\text {min }}=M_{\text {thres }} \approx 0.35$, the $\mathscr{C}_{|u|}^{\text {th }}\left(M_{\text {thres }}\right) \approx 0.26$ value as well as the global shape of $\mathscr{C}_{|u|}^{\text {th }}($.$) are$ retrieved. Yet, as $M_{\text {min }}$ falls below $M_{\text {thres }}$, the measured stable convective Courant number increases to reach the plateau value $\mathscr{C}_{|u|}=0.43$ which is bigger than the one predicted by the above analysis.

In any case, for this specific test case, $\mathscr{C}_{|u|} \approx 0.26$ or equivalently $\mathscr{C}_{C} \approx 1$ is sufficient to ensure the scheme stability for every Mach number. Thus, the "Mach-uniform" stability property, $\mathscr{C}_{|u|} \approx 1, \forall M_{\min }$, obtained by Zakerzadeh in [16] for the IMEX LP scheme and which can be observed in Figure B.14, is not retrieved for the present method. At a given Mach number, the presence of $\pm \mathscr{E}_{0} c_{C}$ in the eigenvalues of the convective subsystem prevents from reaching the stability condition $\mathscr{C}_{|u|} \approx 1$. However, in the above simple test case, the stable convective Courant number $\mathscr{C}_{C}$ no longer behaves as $O\left(M_{\min }\right)$ as $M_{\min } \rightarrow 0$.

This latter result is absolutely not guaranteed by the time-implicit integration of the stiff part of the IMEX approach and strongly depends on the splitting at stake. Indeed, in [14, 15] several IMEX fractional steps on Euler-like systems are studied. It turns out that the "modified" equation obtained from the order two Taylor expansion of a smooth solution of an IMEX fractional step approach exhibits a diffusive operator which can be written as $\mathbf{D}_{0} \partial_{x x} \mathbf{U}$; with $\mathbf{D}_{0}=$ $\beta \mathscr{C}_{|u|} \mathbf{I}-\left(\mathbf{A}_{0}^{\mathcal{C}}\right)^{2}+\left(\mathbf{A}_{0}^{\mathcal{A}}\right)^{2}+\left[\mathbf{A}_{0}^{C}, \mathbf{A}_{0}^{\mathcal{A}}\right], \beta$ being a coefficient related to the scheme numerical diffusion of the convective sub-step, $\mathbf{A}_{0}^{\mathcal{C}}$ (respectively $\mathbf{A}_{0}^{\mathcal{A}}$ ) the linearized jacobian matrix related to the convective (respectively the acoustic) subsystem, and $\left[\mathbf{A}_{0}^{\mathcal{C}}, \mathbf{A}_{0}^{\mathcal{A}}\right]=\mathbf{A}_{0}^{C} \mathbf{A}_{0}^{\mathcal{A}}-\mathbf{A}_{0}^{\mathcal{A}} \mathbf{A}_{0}^{C}$ being the commutator linked to the splitting. The latter can be viewed as a mathematical operator which couples the two subsystems of the fractional step approach. Hence, even if it is discretized using a time-implicit scheme, the acoustic subsystem can still have an influence on the overall fractional step stability through the commutator which can impact the diffusion (or the anti-diffusion) effect of the modified equation. In Figure B.14, the orange-triangle line labeled "Sp-LP" represents the stable convective Courant number 
obtained with the Lagrange-Projection IMEX approach. As already proven in [16], this scheme is Mach-uniformly stable for $\mathscr{C}_{|u|}=1$.

The next subsection provides an analytical stability analysis in order to compare with the above measured stable Courant numbers.

\section{Appendix B.2. A Von Neumann Stability Analysis}

In order to better understand the shape of the stable Courant number, a von Neumann-like stability analysis based on a linearized version of the IMEX approach has been performed. This strategy is motivated by the fact that, as shown in Figure D.15 of Appendix D, the instability seems to appear in a region where $\rho, u$ and $p$ are constant. The linearization required by the von Neumann analysis is thus justified. However, one should keep in mind that such a method relies on periodic boundary conditions which is not the case here because of the density discontinuity.

Starting at time-step $t^{n}$ with a perturbed constant flow:

$$
\begin{aligned}
& \rho_{i}^{n}=\rho^{0}+\epsilon \rho_{i}^{1, n}, \rho^{0}=\rho_{R}^{0}, \\
& u_{i}^{n}=u^{0}+\epsilon u_{i}^{1, n}, \\
& p_{i}^{n}=p^{0}+\epsilon p_{i}^{1, n},
\end{aligned}
$$

the discrete dynamics of the perturbation is derived when a Rusanov scheme is used to solve the first convective subsystem 2.2). The numerical flux at face $i+1 / 2$ associated to this scheme reads:

$$
\begin{aligned}
& { }_{\mathbf{H}_{\text {rus }}^{n} i+1 / 2}=\frac{\mathbf{F}^{C}\left(\mathbf{U}_{i+1}^{n}\right)+\mathbf{F}^{C}\left(\mathbf{U}_{i}^{n}\right)}{2}-\frac{\left|\lambda_{i+1 / 2}^{n}\right|}{2}\left(\mathbf{U}_{i+1}^{n}-\mathbf{U}_{i}^{n}\right), \\
& \text { with: } \mathbf{F}^{C}(\mathbf{U})=\left[\rho u, \rho u^{2}+\mathscr{E}_{0}^{2} p,\left(\rho e+\mathscr{E}_{0}^{2} p\right) u\right]^{T}, \\
& \text { and: }\left|\lambda_{i+1 / 2}^{n}\right|=\max \left(\left|u_{i+1}^{n}\right|+\left(\mathscr{E}_{0}\right)^{n}\left(c_{C}\right)_{i+1}^{n},\left|u_{i}^{n}\right|+\left(\mathscr{E}_{0}\right)^{n}\left(c_{C}\right)_{i}^{n}\right) .
\end{aligned}
$$

Let us define $\rho_{i}^{n+}=\rho_{i}^{0, n+}+\epsilon \rho_{i}^{1, n+}, u_{i}^{n+}=u_{i}^{0, n+}+\epsilon u_{i}^{1, n+}$, and $p_{i}^{n+}=p_{i}^{0, n+}+\epsilon p_{i}^{1, n+}$ the solution produced by the Rusanov scheme applied to the convective subsystem. Then, zeroth order and first order dynamics can be decoupled and one obtains:

$$
\begin{aligned}
& \rho_{i}^{0, n+}=\rho^{0}, \\
& u_{i}^{0, n+}=u^{0}, \\
& p_{i}^{0, n+}=p^{0}, \\
& \frac{\rho_{i}^{1, n+}-\rho_{i}^{1, n}}{\Delta t}+u^{0} \frac{\rho_{i+1}^{1, n}-\rho_{i-1}^{1, n}}{2 \Delta x}+\rho^{0} \frac{u_{i+1}^{1, n}-u_{i-1}^{1, n}}{2 \Delta x}-\left|\lambda^{0}\right| \frac{\rho_{i+1}^{1, n}-2 \rho_{i}^{1, n}+\rho_{i-1}^{1, n}}{2 \Delta x}=0, \\
& \frac{u_{i}^{1, n+}-u_{i}^{1, n}}{\Delta t}+u^{0} \frac{u_{i+1}^{1, n}-u_{i-1}^{1, n}}{2 \Delta x}+\left(\left(\mathscr{E}_{0}\right)^{2} / \rho^{0}\right) \frac{p_{i+1}^{1, n}-p_{i-1}^{1, n}}{2 \Delta x}-\left|\lambda^{0}\right| \frac{u_{i+1}^{1, n}-2 u_{i}^{1, n}+u_{i-1}^{1, n}}{2 \Delta x}=0, \\
& \frac{p_{i}^{1, n+}-p_{i}^{1, n}}{\Delta t}+u^{0} \frac{p_{i+1}^{1, n}-p_{i-1}^{1, n}}{2 \Delta x}+\rho^{0}\left(c_{C}^{0}\right)^{2} \frac{u_{i+1}^{1, n}-u_{i-1}^{1, n}}{2 \Delta x}-\left|\lambda^{0}\right| \frac{p_{i+1}^{1, n}-2 p_{i}^{1, n}+p_{i-1}^{1, n}}{2 \Delta x}=0, \\
& \text { with: }\left|\lambda^{0}\right|=\left|u^{0}\right|+\left(\mathscr{E}_{0}\right)^{n}\left(c_{C}^{0}\right) .
\end{aligned}
$$

If $\forall \phi \in\{\rho, u, p\}$ the following sinus spatial pulse is conjectured $\phi_{i}^{t}=\hat{\phi}^{t} e^{j k x_{i}}, t \in\{n, n+\}$; one can derive the von Neumann gain matrix related to the convective subsystem:

$$
\begin{aligned}
& {\left[\begin{array}{l}
\hat{\rho}^{n+} \\
\hat{u}^{n+} \\
\hat{p}^{n+}
\end{array}\right]=\left[\begin{array}{ccc}
G & -\frac{\rho^{0} \mathscr{C}_{|u|} j \sin (k \Delta x)}{\left|u^{0}\right|} & 0 \\
0 & G & -\frac{\left(\mathscr{E}_{0}^{n}\right)^{2} \mathscr{C}_{|u|}}{\rho^{0}\left|u^{0}\right|} j \sin (k \Delta x) \\
0 & -\frac{\rho^{0}\left(c_{C}^{0}\right)^{2} \mathscr{C}_{|u|}}{\left|u^{0}\right|} j \sin (k \Delta x) & G
\end{array}\right]\left[\begin{array}{l}
\hat{\rho}^{n} \\
\hat{u}^{n} \\
\hat{p}^{n}
\end{array}\right],} \\
& \text { with: } G=1-2 \frac{\left|\lambda^{0}\right|}{\left|u^{0}\right|} \mathscr{C}_{|u|} \sin ^{2}(k \Delta x / 2)-j \frac{u^{0}}{\left|u^{0}\right|} \mathscr{C}_{|u|} \sin (k \Delta x) .
\end{aligned}
$$


The spectral radius $|G|_{C}$ related to this gain matrix can be found easily and one can state that (See Appendix C for a proof):

$$
\mathscr{C}_{|u|}<\mathscr{C}_{|u|}^{\text {crit }}=\left|u^{0}\right| /\left|\lambda^{0}\right| \Rightarrow|G|_{C}<1 .
$$

In particular, when $M_{\text {min }}$ tends towards one, $\left(\mathscr{E}_{0}\right)^{n}$ tends towards one and $c_{C}^{0}$ tends towards $c^{0}$ such that $|G|_{C}<1 \Leftrightarrow \mathscr{C}_{|u|}<$ $M_{\min } /\left(1+M_{\min }\right)$. Thus, the classical CFL condition is retrieved.

The same kind of analysis is performed on the acoustic subsystem. However, the time-implicit flux (4.22) raises new issues in the sense that its energy contribution strongly couples zeroth-order and first-order terms: $\left(p_{\mathcal{A}}^{*}\right)_{i+1 / 2}^{n+1}\left(u_{\mathcal{A}}^{*}\right)_{i+1 / 2}^{n+1}=\left(p_{\mathcal{A}}^{0, *}\right)_{i+1 / 2}^{n+1}\left(u_{\mathcal{A}}^{0, *}\right)_{i+1 / 2}^{n+1}+\epsilon\left(\left(p_{\mathcal{A}}^{0, *}\right)_{i+1 / 2}^{n+1}\left(u_{\mathcal{A}}^{1, *}\right)_{i+1 / 2}^{n+1}+\left(p_{\mathcal{A}}^{1, *}\right)_{i+1 / 2}^{n+1}\left(u_{\mathcal{A}}^{0, *}\right)_{i+1 / 2}^{n+1}\right)$. What is more, this coupling is strongly non-linear and prevents from deriving a simple von Neumann analysis. That is why, it has been additionally assumed that zeroth-order terms including these taken at time $t^{n+1}$ were constant namely:

$$
\begin{aligned}
& \rho_{i}^{0, n+1}=\rho^{0}, \\
& u_{i}^{0, n+1}=u^{0}, \\
& p_{i}^{0, n+1}=p^{0}
\end{aligned}
$$

then, as proved in Appendix C the dynamics of the perturbation reads:

$$
\begin{aligned}
& \frac{\rho_{i}^{1, n+1}-\rho_{i}^{1, n+}}{\Delta t}=0, \\
& \frac{u_{i}^{1, n+1}-u_{i}^{1, n+}}{\Delta t}+\left(1-\left(\mathscr{E}_{0}^{n}\right)^{2}\right)\left[\frac{1}{\rho^{0}} \frac{p_{i+1}^{1, n+1}-p_{i-1}^{1, n+1}}{2 \Delta x}-\frac{a_{\mathcal{H}}^{0}}{\rho^{0}} \frac{u_{i+1}^{1, n+1}-2 u_{i}^{1, n+1}+u_{i-1}^{1, n+1}}{2 \Delta x}\right]=0, \\
& \frac{p_{i}^{1, n+1}-p_{i}^{1, n+}}{\Delta t}+\left(1-\left(\mathscr{E}_{0}^{n}\right)^{2}\right) \rho^{0}\left(c_{\mathcal{A}}^{0}\right)^{2}\left[\frac{u_{i+1}^{1, n+1}-u_{i-1}^{1, n+1}}{2 \Delta x}-\frac{1}{a_{\mathcal{H}}^{0}} \frac{p_{i+1}^{1, n+1}-2 p_{i}^{1, n+1}+p_{i-1}^{1, n+1}}{2 \Delta x}\right]=0 .
\end{aligned}
$$

The von Neumann gain matrix writes:

$$
\begin{aligned}
& {\left[\begin{array}{ccc}
1 & 0 & 0 \\
0 & 1+2 \alpha^{n} \sin ^{2}(k \Delta x / 2) & \frac{\alpha^{n}}{a_{\mathcal{P}}^{0}} j \sin (k \Delta x) \\
0 & \alpha^{n}\left(\omega_{\mathcal{A}}^{0}\right)^{2} a_{\mathcal{A}}^{0} j \sin (k \Delta x) & 1+2 \alpha^{n}\left(\omega_{\mathcal{A}}^{0}\right)^{2} \sin ^{2}(k \Delta x / 2)
\end{array}\right]\left[\begin{array}{l}
\hat{\rho}^{n+1} \\
\hat{u}^{n+1} \\
\hat{p}^{n+1}
\end{array}\right]=\left[\begin{array}{l}
\hat{\rho}^{n+} \\
\hat{u}^{n+} \\
\hat{p}^{n+}
\end{array}\right],} \\
& \text { with: } \alpha^{n}=\left(1-\left(\mathscr{E}_{0}\right)^{2}\right) \frac{a_{\mathcal{A}}^{0} \mathscr{C}_{|u|}}{\rho^{0}\left|u^{0}\right|} \\
& \text { and: } \omega_{\mathcal{A}}^{0}=\frac{\rho^{0} c_{\mathcal{A}}^{0}}{a_{\mathcal{A}}^{0}}
\end{aligned}
$$

Finally the von Neumann gain matrix related to the fractional step approach reads:

$$
\left[\begin{array}{lc}
G & 0 \\
0 & \mathbf{G}_{\mathcal{A}}^{-1} \mathbf{G}_{C}
\end{array}\right]
$$

with:

$$
\begin{aligned}
& \mathbf{G}_{C}=\left[\begin{array}{cc}
G & -\frac{\left(\mathscr{E}_{0}^{n}\right)^{2} \mathscr{C}_{|u|}}{\rho^{0}\left|u^{0}\right|} j \sin (k \Delta x) \\
-\frac{\rho^{0}\left(c_{C}^{0}\right)^{2} \mathscr{C}_{|u|}}{\left|u^{0}\right|} j \sin (k \Delta x) & G
\end{array}\right], \\
& \mathbf{G}_{\mathcal{A}}=\left[\begin{array}{cc}
1+2 \alpha^{n} \sin ^{2}(k \Delta x / 2) & \frac{\alpha^{n}}{a_{\mathcal{P}}^{0}} j \sin (k \Delta x) \\
\alpha^{n}\left(\omega_{\mathcal{A}}^{0}\right)^{2} a_{\mathcal{A}}^{0} j \sin (k \Delta x) & 1+2 \alpha^{n}\left(\omega_{\mathcal{A}}^{0}\right)^{2} \sin ^{2}(k \Delta x / 2)
\end{array}\right], \\
& \mathbf{G}_{\mathcal{A}}^{-1}=\frac{1}{\Delta}\left[\begin{array}{cc}
1+2 \alpha^{n}\left(\omega_{\mathcal{A}}^{0}\right)^{2} \sin ^{2}(k \Delta x / 2) & -\frac{\alpha^{n}}{a_{\mathcal{P}}^{0}} j \sin (k \Delta x) \\
-\alpha^{n}\left(\omega_{\mathcal{A}}^{0}\right)^{2} a_{\mathcal{P}}^{0} j \sin (k \Delta x) & 1+2 \alpha^{n} \sin ^{2}(k \Delta x / 2)
\end{array}\right], \Delta=\operatorname{det} \mathbf{G}_{\mathcal{A} .}
\end{aligned}
$$

$\mathrm{G}$ is a first eigenvalue related to the mass equation. As written in Appendix $\mathrm{C}|G|<1$ if $\mathscr{C}_{|u|}<\mathscr{C}_{|u|}^{\text {crit }}$. The two remaining eigenvalues are linked to the matrix $\mathbf{G}_{\mathcal{A}}^{-1} \mathbf{G}_{C}$ which couples the momentum and the energy equations and can not be 
easily found analytically. Starting from $\mathscr{C}_{|u|}^{\text {crit }}$, a loop on all the $k$ modes $\in[0,4 \pi / \Delta x]$ is performed and the spectral radius $|G|_{\mathcal{A C}}^{k}$ associated to $\left(\mathbf{G}_{\mathcal{A}}^{-1} \mathbf{G}_{C}\right)(k)$ is calculated. If, during the loop, $|G|_{\mathcal{A C}}^{k}>1$, then $\mathscr{C}_{|u|}^{\text {crit }}$ is slightly decreased and the loop is restarted. Otherwise the current Courant number is stored and considered as the stable Courant number of the above von Neumann analysis.

In Figure B.14, the curve labeled "VN-Sp" (red line) displays the different stable convective Courant numbers obtained thanks to the above von Neumann method. Recall that the analytical curve $M \rightarrow \mathscr{C}_{|u|}^{\text {th }}(M)$ is equivalent to $\mathscr{C}_{C}=1$ for this test case. For $M_{\min } \in\left[7.5 \times 10^{-2}, 1\right]$, "VN-Sp" and $M \rightarrow \mathscr{C}_{|u|}^{\text {th }}(M)$ overlap quasi-perfectly. Unfortunately, for very low Mach numbers, $\mathscr{C}_{|u|}$ plunges down. For example, it predicts $\mathscr{C}_{|u|}=10^{-2}$ when $M_{\min }=10^{-4}$.

This difference could stem from the assumptions made on the time-implicit acoustic scheme in order to linearize it. Indeed the zeroth order terms of the updated solution have been assumed to be constant:

$$
\begin{aligned}
& \rho_{i}^{0, n+1}=\rho^{0}, \\
& u_{i}^{0, n+1}=u^{0}, \\
& p_{i}^{0, n+1}=p^{0} .
\end{aligned}
$$

The resulting dynamics then binds only first-order pressure terms with first-order velocity terms. Yet, in that case the small perturbation parameter $\epsilon$ is not related to the flow Mach number. As formally shown in [19, 20] on the continuous subsystem $\mathcal{A}$, one could make the discrete acoustic scheme (4.22) non-dimensional and consider a constant base flow perturbed by modes written in powers of the Mach number. Then, one would observe that the zeroth-order momentum term is fed by the second-order pressure gradient. Thus, one could assume that if the amplitude of this second-order pressure gradient explodes because of an instability, the zeroth-order momentum term would rise too. It would result in an increase of the Mach number associated with a decrease of the discrete numerical time-step built on the zerothorder velocity terms. This might damp the instability. In any case, further investigations should be done in order to better understand this sudden drop.

\section{Appendix C. Study of the von Neumann Gain Matrices}

This appendix is dedicated to the study of the von Neumann gain matrices written in (B.8) and (B.13). The derivation of the convective gain matrix being relatively straightforward, special attention is paid to the expression of its eigenvalues as well as a sufficient condition ensuring that their modulus is strictly lower than one. Besides, the derivation of the acoustic gain matrix is completely done.

\section{Appendix C.1. Analysis of the Convective Gain Matrix Eigenvalues}

Let us consider the von Neumann gain matrix associated with the convective sub-step:

$$
\begin{aligned}
& {\left[\begin{array}{ccc}
G & -\frac{\rho^{0} \mathscr{C}_{|u|}}{\left|u^{0}\right|} j \sin (k \Delta x) & 0 \\
0 & G & -\frac{\left(\mathscr{E}_{0}^{n}\right)^{2} \mathscr{C}_{|u|}}{\rho^{0}\left|u^{0}\right|} j \sin (k \Delta x) \\
0 & -\frac{\rho^{0}\left(c_{C}^{0}\right)^{2} \mathscr{C}_{|u|}}{\left|u^{0}\right|} j \sin (k \Delta x) & G
\end{array}\right],} \\
& \text { and: } G=1-2 \frac{\mathscr{C}_{|u|}}{\mathscr{C}_{|u|}^{\text {crit }}} \sin ^{2}(k \Delta x / 2)-j \frac{u^{0}}{\left|u^{0}\right|} \mathscr{C}_{|u|} \sin (k \Delta x), \\
& \text { with: } \left.\mathscr{C}_{|u|}^{\text {crit }}=\left|u^{0}\right| /\left|\lambda^{0}\right| \in\right] 0,1[.
\end{aligned}
$$


$G$ is the first eigenvalue of this matrix. Define $X=\sin ^{2}(k \Delta x / 2) \in[0,1]$, then $\sin ^{2}(k \Delta x)=4 X(1-X)$. Thus:

$$
\begin{gathered}
|G|^{2}=\left(1-2 \frac{\mathscr{C}_{|u|}}{\mathscr{C}_{|u|}^{\text {crit }}} X\right)^{2}+4 \mathscr{C}_{|u|}^{2} X(1-X), \\
|G|^{2}<1 \Leftrightarrow \mathscr{C}_{|u|}<\frac{\mathscr{C}_{|u|}^{\text {crit }}}{\left(\left(1-\left(\mathscr{C}_{|u|}^{\text {crit }}\right)^{2}\right) X+\left(\mathscr{C}_{|u|}^{\text {crit }}\right)^{2}\right)} .
\end{gathered}
$$

498 Since $X \in[0,1]$ the most constraining CFL condition is $\mathscr{C}_{|u|}<\mathscr{C}_{|u|}^{\text {crit }}$.

The two other eigenvalues of the above gain matrix are the roots of the characteristic polynomial function:

$$
(G-\lambda)^{2}+\left(\mathscr{E}_{0}\right)^{2}\left(\mathscr{C}_{|u|}\right)^{2}\left(\frac{c_{C}^{0}}{u^{0}}\right)^{2} \sin ^{2}(k \Delta x)
$$

They write:

$$
\begin{aligned}
& \lambda^{ \pm}=G \mp j \mathscr{E}_{0}^{n} \mathscr{C}_{|u|}\left|\frac{c_{\mathcal{C}}^{0}}{u^{0}}\right| \sin (k \Delta x) \mid, \\
& \left|\lambda^{ \pm}\right|^{2}=\left(1-2 \frac{\mathscr{C}_{|u|}}{\mathscr{C}_{|u|}^{\text {crit }}} X\right)^{2}+\mathscr{C}_{|u|}^{2}\left(\left[1+\left(\mathscr{E}_{0}^{n}\right)^{2}\left|\frac{c_{\mathcal{C}}^{0}}{u^{0}}\right|^{2}\right] 4 X(1-X) \pm 2 \mathscr{E}_{0} \frac{u^{0}}{\left|u^{0}\right|}\left|\frac{c_{\mathcal{C}}^{0}}{u^{0}}\right| \sin (k \Delta x)|\sin (k \Delta x)|\right) \\
& \Rightarrow\left|\lambda^{ \pm}\right|^{2} \leq\left(1-2 \frac{\mathscr{C}_{|u|}}{\mathscr{C}_{|u|}^{\text {crit }}} X\right)^{2}+4\left(1+\mathscr{E}_{0}\left|\frac{c_{\mathcal{C}}^{0}}{u^{0}}\right|\right)^{2} \mathscr{C}_{|u|}^{2} X(1-X)=\left(1-2 \frac{\mathscr{C}_{|| u \mid}}{\mathscr{C}_{|u|}^{\text {crit }}} X\right)^{2}+4\left(\frac{\mathscr{C}_{|| u \mid}}{\mathscr{C}_{|u|} \text { crit }}\right)^{2} X(1-X) .
\end{aligned}
$$

A sufficient condition ensuring that $\left|\lambda^{ \pm}\right|<1$ is once again $\mathscr{C}_{|u|}<\mathscr{C}_{|u|}^{\text {crit }}$.

\section{Appendix C.2. Derivation of the Linearized Acoustic Dynamics}

The von Neumann analysis has to be made on the relaxation system (3.9). Define at time $t^{n+}$ :

$$
\begin{aligned}
& W_{i}^{n+}=u_{i}^{n+}-\frac{p_{i}^{n+}}{a_{\mathcal{A}}^{n+}}=u_{i}^{n+}-\frac{\Pi_{i}^{n+}}{a_{\mathcal{A}}^{n+}}, \\
& R_{i}^{n+}=u_{i}^{n+}+\frac{p_{i}^{n+}}{a_{\mathcal{A}}^{n+}}=u_{i}^{n+}+\frac{\Pi_{i}^{n+}}{a_{\mathcal{A}}^{n+}} .
\end{aligned}
$$

Then,

$$
\begin{aligned}
& \left\{\begin{array}{l}
\frac{\rho_{i}^{n+1}-\rho_{i}^{n+}}{\Delta t}=0, \\
\frac{(\rho u)_{i}^{n+1}-(\rho u)_{i}^{n+}}{\Delta t}+\left(1-\left(\mathscr{E}_{0}^{n}\right)^{2}\right) \frac{\left(p_{\mathcal{A}}^{*}\right)_{i+1 / 2}^{n+1}-\left(p_{\mathcal{P}}^{*}\right)_{i-1 / 2}^{n+1}}{\Delta x}=0, \\
\frac{(\rho \Pi)_{i}^{n+1}-(\rho \Pi)_{i}^{n+}}{\Delta t}+\left(1-\left(\mathscr{E}_{0}^{n}\right)^{2}\right)\left(a_{\mathcal{A}}^{n+}\right)^{2} \frac{\left(u_{\mathcal{A}}^{*}\right)_{i+1 / 2}^{n+1}-\left(u_{\mathcal{H}}^{*}\right)_{i-1 / 2}^{n+1}}{\Delta x}=0, \\
\frac{(\rho e)_{i}^{n+1}-(\rho e)_{i}^{n+}}{\Delta t}+\left(1-\left(\mathscr{E}_{0}^{n}\right)^{2}\right) \frac{\left(p_{\mathcal{A}}^{*} u_{\mathcal{P}}^{*}\right)_{i+1 / 2}^{n+1}-\left(p_{\mathcal{A}}^{*} u_{\mathcal{A}}^{*}\right)_{i-1 / 2}^{n+1}}{\Delta x}=0,
\end{array}\right. \\
& \Leftrightarrow \\
& \begin{cases}\frac{\rho_{i}^{n+1}-\rho_{i}^{n+}}{\Delta t} & =0, \\
\frac{(\rho u)_{i}^{n+1}-(\rho u)_{i}^{n+}}{\Delta t} & +\left(1-\left(\mathscr{E}_{0}^{n}\right)^{2}\right)\left[\frac{\Pi_{i+1}^{n+1}-\Pi_{i-1}^{n+1}}{2 \Delta x}-\frac{a_{\mathcal{P}}^{n+}}{2} \frac{u_{i+1}^{n+1}-2 u_{i}^{n+1}+u_{i-1}^{n+1}}{\Delta x}\right]=0, \\
\frac{(\rho \Pi)_{i}^{n+1}-(\rho \Pi)_{i}^{n+}}{\Delta t} & +\left(1-\left(\mathscr{E}_{0}\right)^{2}\right)\left[\left(a_{\mathcal{A}}^{n+}\right)^{2} \frac{u_{i+1}^{n+1}-u_{i-1}^{n+1}}{2 \Delta x}-\frac{a_{\mathcal{P}}^{n+}}{2} \frac{\Pi_{i+1}^{n+1}-2 \Pi_{i}^{n+1}+\Pi_{i-1}^{n+1}}{\Delta x}\right]=0, \\
\frac{(\rho e)_{i}^{n+1}-(\rho e)_{i}^{n+}}{\Delta t} & +\left(1-\left(\mathscr{E}_{0}^{n}\right)^{2}\right)\left[\frac{(\Pi u)_{i+1}^{n+1}-(\Pi u)_{i-1}^{n+1}}{2 \Delta x}-\frac{1}{4 a_{\mathcal{A}}^{n+}} \frac{\left(\Pi^{2}\right)_{i+1}^{n+1}-2\left(\Pi^{2}\right)_{i}^{n+1}+\left(\Pi^{2}\right)_{i-1}^{n+1}}{\Delta x}\right. \\
& \left.-\frac{a_{\mathcal{P}}^{n+}}{4} \frac{\left(u^{2}\right)_{i+1}^{n+1}-2\left(u^{2}\right)_{i}^{n+1}+\left(u^{2}\right)_{i-1}^{n+1}}{\Delta x}\right]=0 .\end{cases}
\end{aligned}
$$


Supposing that $\forall \phi \in\{\rho, u, \Pi, e\}, \phi_{i}^{0, n+1}=\phi^{0}$ a constant, one can extract the linearized dynamics related to C.6]:

$$
\begin{aligned}
& \frac{\rho_{i}^{1, n+1}-\rho_{i}^{1, n+}}{\Delta t}=0, \\
& \rho^{0} \frac{u_{i}^{1, n+1}-u_{i}^{1, n+}}{\Delta t}+\left(1-\left(\mathscr{E}_{0}^{n}\right)^{2}\right)\left[\frac{\Pi_{i}^{1, n+1}-\Pi_{i}^{1, n+1}}{2 \Delta x}-\frac{a_{\mathcal{F}}^{0}}{2} \frac{u_{i+1}^{1, n+1}-2 u_{i}^{1, n+1}+u_{i-1}^{1, n+1}}{\Delta x}\right]=0, \\
& \rho^{0} \frac{\Pi_{i}^{1, n+1}-\Pi_{i}^{1, n+}}{\Delta t}+\left(1-\left(\mathscr{E}_{0}^{n}\right)^{2}\right)\left[\left(a_{\mathcal{A}}^{0}\right)^{2} \frac{u_{i}^{1, n+1}-u_{i}^{1, n+1}}{2 \Delta x}-\frac{a_{\mathcal{A}}^{0}}{2} \frac{\Pi_{i+1}^{1, n+1}-2 \Pi_{i}^{1, n+1}+\Pi_{i-1}^{1, n+1}}{\Delta x}\right]=0, \\
& \rho^{0} \frac{e_{i}^{1, n+1}-e_{i}^{1, n+}}{\Delta t}+\left(1-\left(\mathscr{E}_{0}^{n}\right)^{2}\right)\left[p^{0} \frac{u_{i}^{1, n+1}-u_{i}^{1, n+1}}{2 \Delta x}+u^{0} \frac{\Pi_{i}^{1, n+1}-\Pi_{i}^{1, n+1}}{2 \Delta x}\right] \\
& -\left(1-\left(\mathscr{E}_{0}^{n}\right)^{2}\right)\left[\frac{p^{0}}{a_{\mathcal{A}}^{0}} \frac{\prod_{i+1}^{1, n+1}-2 \Pi_{i}^{1, n+1}+\Pi_{i-1}^{1, n+1}}{2 \Delta x}+u^{0} a_{\mathcal{A}}^{0} \frac{u_{i+1}^{1, n+1}-2 u_{i}^{1, n+1}+u_{i-1}^{1, n+1}}{2 \Delta x}\right]=0 .
\end{aligned}
$$

During the projection step, $\Pi_{i}^{n+1}=p_{i}^{n+1}=p^{E O S}\left(\rho_{i}^{n+1}, \varepsilon_{i}^{n+1}\right)$ is imposed with $\varepsilon_{i}^{n+1}=e_{i}^{n+1}-\left(u_{i}^{n+1}\right)^{2} / 2$. If one assumes that this projection holds separately for zeroth order and first order terms then $\forall \sharp \in\{n+, n+1\}$ :

$$
\Pi_{i}^{1, \sharp}=p_{i}^{1, \sharp}=\left(\partial_{\rho} p_{\mid \varepsilon}\right)^{0} \rho_{i}^{1, \sharp}+\left(\partial_{\varepsilon} p_{\mid \rho}\right)^{0}\left(e_{i}^{1, \sharp}-u^{0} u_{i}^{1, \sharp}\right) .
$$

Using the momentum equation, the perturbed pressure dynamics is then:

$$
\left.\frac{p_{i}^{1, n+1}-p_{i}^{1, n+}}{\Delta t}+\left(1-\left(\mathscr{E}_{0}^{n}\right)^{2}\right)\left(\partial_{\varepsilon} p_{\rho}\right)\right)^{0} \frac{p^{0}}{\rho^{0}}\left[\frac{u_{i+1}^{1, n+1}-u_{i-1}^{1, n+1}}{2 \Delta x}-\frac{1}{a_{\mathcal{F}}^{0}} \frac{p_{i+1}^{1, n+1}-2 p_{i}^{1, n+1}+p_{i-1}^{1, n+1}}{2 \Delta x}\right]=0 .
$$

According to definition $\left[2.6,\left(\partial_{\varepsilon} p_{\rho}\right)\right)^{0} \frac{p^{0}}{\rho^{0}}$ is exactly equal to $\rho^{0}\left(c_{\mathcal{P}}^{0}\right)^{2}$. The linearized dynamics of the non conservative variables related to the acoustic scheme is then:

$$
\begin{aligned}
& \frac{\rho_{i}^{1, n+1}-\rho_{i}^{1, n+}}{\Delta t}=0, \\
& \frac{u_{i}^{1, n+1}-u_{i}^{1, n+}}{\Delta t}+\left(1-\left(\mathscr{E}_{0}^{\mathscr{n}}\right)^{2}\right)\left[\frac{1}{\rho^{0}} \frac{p_{i+1}^{1, n+1}-p_{i-1}^{1, n+1}}{2 \Delta x}-\frac{a_{\mathcal{P}}^{0}}{\rho^{0}} \frac{u_{i+1}^{1, n+1}-2 u_{i}^{1, n+1}+u_{i-1}^{1, n+1}}{2 \Delta x}\right]=0, \\
& \frac{p_{i}^{1, n+1}-p_{i}^{1, n+}}{\Delta t}+\left(1-\left(\mathscr{E}_{0}\right)^{2}\right) \rho^{0}\left(c_{\mathcal{F}}^{0}\right)^{2}\left[\frac{u_{i+1}^{1, n+1}-u_{i-1}^{1, n+1}}{2 \Delta x}-\frac{1}{a_{\mathcal{H}}^{0}} \frac{p_{i+1}^{1, n+1}-2 p_{i}^{1, n+1}+p_{i-1}^{1, n+1}}{2 \Delta x}\right]=0 .
\end{aligned}
$$

\section{Appendix D. Location of the IMEX Instability}

Figure D.15 and Figure D.16 show the growth of the numerical instability observed in the case presented in subsection 5.1.2. The picture is taken at time $t=2.496 \times 10^{-2} s$ but for a mesh of $10^{3}$ cells (Figure D.15) and for a finer one of $5 \times 10^{3}$ cells (Figure D.16).

One can observe than the instability originates from the region located after the contact discontinuity front where the Mach number takes its lowest value. As the mesh is refined, the amplitude of the instability surges considerably since the numerical diffusion is largely diminished. 


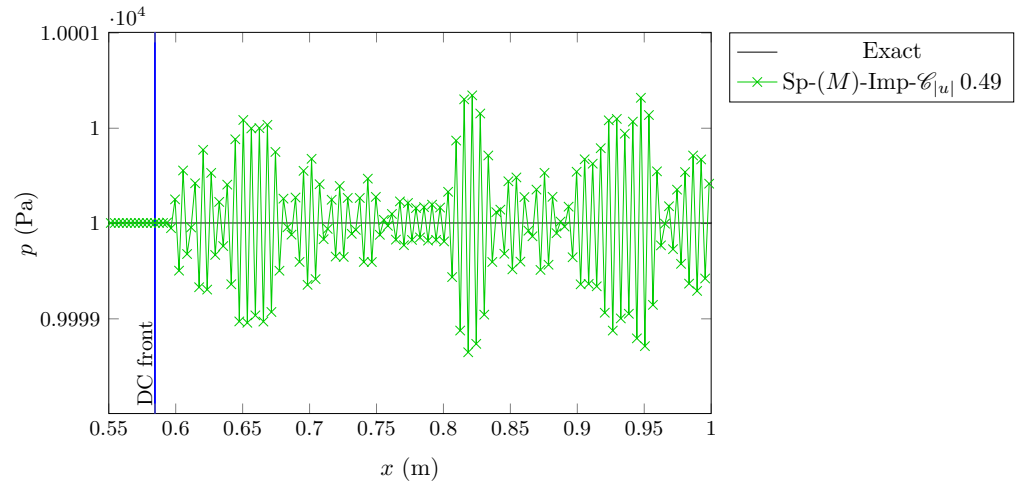

Figure D.15: $p$, Ideal Gas, $M_{\text {min }}=10^{-2}$, with $\mathbf{N}_{\text {cells }}=\mathbf{1 0}^{\mathbf{3}}, \mathscr{C}_{|u|}=0.49$, iteration $270,\left(t=2.496 \times 10^{-2} s\right)$

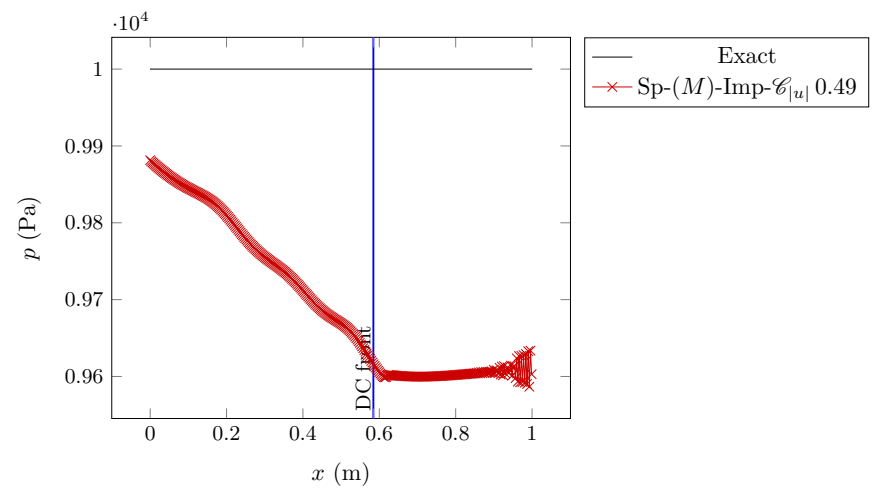

Figure D.16: $p$, Ideal Gas, $M_{\min }=10^{-2}$, with $\mathbf{N}_{\text {cells }}=\mathbf{5} \times \mathbf{1 0}^{\mathbf{3}}, \mathscr{C}_{|u|}=0.49$, iteration $1399,\left(t=2.497 \times 10^{-2} s\right)$ 


\section{Appendix E. The Most Constraining Euler Courant Number}

Let us consider the shock tube test case presented in subsection 5.2 The fastest Euler eigenvalue is $u^{*}+c_{R}^{0, *}$ with $c_{R}^{0, *}=c\left(\rho_{R}^{*}, p^{*}\right)$. It corresponds to the characteristic colliding with the 3-shock wave front speed. Here $u^{*}$ and $p^{*}$ are the intermediate velocity and pressure whose values can be approximatively calculated: $u^{*} \approx 1.49886 \mathrm{~m} \mathrm{~s}^{-1}$, $p^{*} \approx 10020.9 P a$. What is more, the conservation of entropy through the 3-shock brings: $\rho_{R}^{*}=\rho_{R}^{0}\left(p^{*} / p_{R}^{0}\right)^{1 / \gamma}$, and $c\left(\rho_{R}^{*}, p^{*}\right)=c_{R}^{0}\left(p^{*} / p_{R}^{0}\right)^{(\gamma-1) / \gamma} \approx 336.36256 m s^{-1}$.

The most constraining time-step got from the above wave speed writes:

$$
\Delta t_{E}^{0, *}=\frac{\mathscr{C}_{E}^{0, *}}{2} \frac{\Delta x}{u^{*}+c_{R}^{0, *}} .
$$

Besides the time-step related to $u^{0}$ writes simply $\Delta t_{C}^{u^{0}}=\mathscr{C}_{|u|}^{u^{0}} \Delta x / u^{0}$. Then:

$$
\Delta t_{C}^{u^{0}}=\Delta t_{E}^{0, *} \Leftrightarrow \mathscr{C}_{|u|}^{u^{0}}=\frac{u^{0}}{2\left(u^{*}+c_{R}^{0, *}\right)} \mathscr{C}_{E}^{0, *} \approx 1.48649 \times 10^{-3} \mathscr{C}_{E}^{0, *} .
$$




\section{References}

[1] N. E. Joukowski, Memoirs of the Imperial Academy Society of St. Petersburg, Proceedings of the American Water Works Association 24 (1898) 341-424.

[2] L. Allievi, Teoria generale del moto perturbato dell'acqua nei tubi in pressione (coplo d'ariete), Tip. del Genio civile . (1903) .

[3] M. S. Ghidaoui, M. Zhao, D. A. McInnis, D. H. Axworthy, A review of water hammer theory and practice, Applied Mechanics Reviews 58 (2005) 49.

[4] A. R. Simpson, E. B. Wylie, Large water-hammer pressures for column separation in pipelines, Journal of Hydraulic Engineering 117 (1991) 1310-1316.

[5] E. Turkel, Preconditioned methods for solving the incompressible and low speed compressible equations, Journal of Computational Physics 72 (1987) 277-298.

[6] H. Guillard, C. Viozat, On the behavior of upwind schemes in the low Mach number limit, Computers and Fluids 28 (1999) 63-86.

[7] H. Guillard, A. Murrone, On the behavior of upwind schemes in the low Mach number limit: II Godunov type schemes, Computers and Fluids 33 (2004) 655-675.

[8] A. Murrone, H. Guillard, Behavior of upwind scheme in the low Mach number limit: III. Preconditioned dissipation for a five equation two phase model, Computers and Fluids 37(10) (2008) 1209-1224.

[9] S. Jin, Efficient asymptotic-preserving (AP) schemes for some multiscale kinetic equations, SIAM Journal on Scientific Computing 21 (1999) 441-454.

[10] P. Degond, M. Tang, All speed scheme for the low Mach number limit of the isentropic Euler equation, Communications in Computational Physics 10 (2011) 1-31.

[11] J. Haack, S. Jin, J. G. Liu, An all-speed asymptotic-preserving method for the isentropic Euler and Navier-Stokes equations, Communications in Computational Physics 12 (2012) 955-980.

[12] S. Noelle, G. Bispen, K. R. Arun, M. Lukáčová-Medvid'ová, C. D. Munz, A weakly asymptotic preserving low Mach number scheme for the Euler equations of gas dynamics, SIAM Journal on Scientific Computing 36 (2014) B989-B1024.

[13] G. Dimarco, R. Loubère, M.-H. Vignal, Study of a new asymptotic preserving scheme for the Euler system in the low Mach number limit, SIAM: Journal of Scientific Computing 39 (2017) 2099-2128.

[14] J. Schütz, S. Noelle, Flux splitting for stiff equations: a notion on stability, Journal of Scientific Computing 64 (2015) 522-540.

[15] H. Zakerzadeh, S. Noelle, A note on the stability of implicit-explicit flux splittings for stiff hyperbolic systems, Preprint IGPM https://www.igpm.rwth-aachen.de/forschung/preprints/449 (2016) .

[16] H. Zakerzadeh, On the Mach-uniformity of the Lagrange-Projection scheme, ESAIM: Mathematical Modelling and Numerical Analysis 51 (2017) 1343-1366.

[17] C. Chalons, M. Girardin, S. Kokh, An all-regime Lagrange-Projection like scheme for the gas dynamics equations on unstructured meshes, Communications in Computational Physics 20 (2016) 188-233.

[18] F. Coquel, Q. L. Nguyen, M. Postel, Q. H. Tran, Entropy-satisfying relaxation method with large time-steps for Euler IBVPS, Mathematics of Computation 79 (2010) 1493-1533.

[19] D. Iampietro, F. Daude, P. Galon, J. M. Hérard, A Mach-sensitive splitting approach for Euler-like systems, https://hal.archivesouvertes.fr/hal-01466827 (2017).

[20] D. Iampietro, F. Daude, P. Galon, J. M. Hérard, A Mach-sensitive splitting approach for Euler-like systems, ESAIM: Mathematical Modelling and Numerical Analysis Accepted for publication (2017).

[21] R. Baraille, G. Bourdin, F. Dubois, A. Y. Le Roux, Une version à pas fractionnaires du schéma de Godunov pour l'hydrodynamique, Compte Rendu de l'Académie des Sciences 314 (1992) 147-152.

[22] T. Buffard, J.-M. Hérard, A conservative fractional step method to solve non-isentropic Euler equations, Computer Methods in Applied Mechanics and Engineering 144 (1996) 199-225.

[23] I. Suliciu, On the thermodynamics of fluids with relaxation and phase transitions, International Journal of Engineering Science 36 (1998) 921-947.

[24] F. Bouchut, Nonlinear Stability of Finite Volume Methods for Hyperbolic Conservation Laws, Birkäser, 2004.

[25] F. Coquel, E. Godlewski, N. Seguin, Relaxation of fluid systems, Mathematical Models and Methods in Applied Science 22 (2012) 43-95.

[26] S. Clerc, Numerical simulation of the homogeneous equilibrium model for two-phase flows, Journal of Computational Physics 161 (2000) 354-375.

[27] F. Bouchut, Entropy satisfying flux vector splittings and kinetic BGK models, Numerische Mathematik 94 (2003) 623-672.

[28] J. B. Whitham, Linear and Non Linear Waves, John Wiley \& Sons Inc, 1974.

[29] M. Girardin, Asymptotic preserving and all-regime Lagrange-Projection like numerical schemes: application to two-phase flows in low Mach regime, Ph.D. thesis, Université Pierre et Marie Curie, https://tel.archives-ouvertes.fr/tel-01127428 (2015).

[30] T. Y. Hou, P. G. Le Floch, Why nonconservative schemes converge to wrong solutions: error analysis, Mathematics of Computation 62 (1994) 497-530.

[31] T. Gallouët, J.-M. Hérard, N. Seguin, A hybrid scheme to compute contact discontinuities in one-dimensional Euler systems., ESAIM: Mathematical Modelling and Numerical Analysis 36 (2002) 1133-1159.

[32] G. R. McGuire, J. L. Morris, A class of implicit, second-order accurate, dissipative schemes for solving systems of conservation laws, Journal of Computational Physics 14 (1974) 126-147.

[33] G. R. McGuire, J. L. Morris, Explicit-implicit schemes for the numerical solution of nonlinear hyperbolic systems, Mathematics of Computation 29 (1975) 407-424.

[34] W. F. Ballhaus, P. M. Goorjian, Implicit finite-difference computations of unsteady transonic flows about airfoils, AIAA Journal 15 (1977) $1728-1735$.

[35] W. F. Ballhaus, P. M. Goorjian, Implicit methods of second-order accuracy for the Euler equations, AIAA Journal 23 (1985) 33-40. 
[36] M. P. Martín, G. V. Candler, A parallel implicit method for the direct numerical simulation of wall-bounded compressible turbulence, Journal of Computational Physics 215 (2006) 153-171.

[37] F. Daude, I. Mary, P. Comte, Self-adaptative Newton-based iteration strategy for the LES of turbulent multi-scale flows, Computers and Fluids 100 (2014) 278-290.

[38] C. Chalons, M. Girardin, S. Kokh, An all-regime Lagrange-Projection like scheme for 2D homogeneous models for two-phase flows on unstructured meshes, Journal of Computational Physics 335 (2016) 885-904.

[39] S. Dellacherie, P. Omnes, J. Jung, P. Raviart, Construction of modified Godunov type schemes accurate at any Mach number for the compressible Euler system, Mathematical Models and Methods in Applied Science 26 (2016) 2525-2615.

[40] R. Klein, Semi-implicit extension of a Godunov-type scheme based on low Mach number asymptotics I: One-dimensional flow, Journal of Computational Physics 121 (1995) 213-237. 